\title{
Diffusion Driven Oscillations in Gene Regulatory Networks
}

\author{
Cicely K Macnamara*, Mark AJ Chaplain \\ School of Mathematics and Statistics, Mathematical Institute, University of St Andrews, United Kingdom, KY16 9SS
}

\begin{abstract}
Gene regulatory networks (GRNs) play an important role in maintaining cellular function by correctly timing key processes such as cell division and apoptosis. GRNs are known to contain similar structural components, which describe how genes and proteins within a network interact - typically by feedback. In many GRNs, proteins bind to gene-sites in the nucleus thereby altering the transcription rate. If the binding reduces the transcription rate there is a negative feedback leading to oscillatory behaviour in mRNA and protein levels, both spatially (e.g. by observing fluorescently labelled molecules in single cells) and temporally (e.g. by observing protein/mRNA levels over time). Mathematical modelling of GRNs has focussed on such oscillatory behaviour. Recent computational modelling has demonstrated that spatial movement of the molecules is a vital component of GRNs, while it has been proved rigorously that the diffusion coefficient of the protein/mRNA acts as a bifurcation parameter and gives rise to a Hopf-bifurcation. In this paper we consider the spatial aspect further by considering the specific location of gene and protein production, showing that there is an optimum range for the distance between an mRNA gene-site and a protein production site in order to achieve oscillations. We first present a model of a well-known GRN, the Hes1 system, and then extend the approach to examine spatio-temporal models of synthetic GRNs e.g. n-gene repressilator and activator-repressor systems. By incorporating the idea of production sites into such models we show that the spatial component is vital to fully understand GRN dynamics.
\end{abstract}

Keywords: Hes1 protein, synthetic networks, repressilators, activator-repressor systems, spatial modelling

\section{Introduction}

A gene regulatory network (GRN) can be defined as a collection of DNA segments in a cell which interact with each other indirectly through their RNA and protein products. GRNs lie at the heart of intracellular signal transduction and indirectly control many important cellular functions such as cell division, apoptosis and adhesion. One key class of GRNs is a group of proteins called transcription factors. As the name suggests, in response to a range of signals, transcription factors change the transcription rate of genes, allowing cells to alter the levels of proteins they require at any given time. A GRN is said to contain a negative feedback loop if a gene product inhibits its own production either

\footnotetext{
*Corresponding author. Phone: +44 (0)1334 463723

Email addresses: ckm@st-andrews.ac.uk (Cicely K Macnamara), majc@st-andrews.ac.uk (Mark AJ Chaplain)
}

15 directly or indirectly, and similarly, is said to contain ${ }_{16}$ a positive feedback loop if a gene product enhances its own production either directly or indirectly. In particular, the modification of the transcription of genes by proteins (transcription factors) through negative feedback (down-regulation) is an important component of many gene networks, and such negative feedback systems are known to exhibit oscillations in the levels of the molecules involved. Negative feedback loops are commonly found in diverse biological processes including inflammation, meiosis, apoptosis and the heat shock response (Lahav et al., 2004), where the oscillatory expression is of particular importance. In addition to their natural occurrence, GRNs have also become an important focus in the emerging field of synthetic biology. Since the pioneering work of Becskei and Serrano (2000) and Elowitz and Leibler (2000), there has been a great deal of interest in synthetic GRNs, both from a practical, experimental viewpoint (Balagadde et al., 2008; Chen et al., 2012; Yordanov et al., 2014) 
and from a theoretical, modelling viewpoint (Purcell ${ }_{87}$ et al., 2010; O’Brien et al., 2012).

Mathematical modelling of GRNs can be traced back 50 years to the seminal paper of Goodwin (1965), followed shortly after by the paper of Griffith (1968). These papers proposed a generic "closed-loop" negative feedback model for a simple mRNA-protein feedback system (which we note is appropriate to model the actual Hes 1 protein system, Hirata et al. (2002)). The models were restricted to purely temporal ODEs and oscillatory behaviour was elusive. Mackey and Glass (1977) introduced the idea of incorporating delays into differential equations. Delay-differential equation mod- 100 els for GRNs have been studied extensively for the last ${ }_{101}$ two decades, since the early work of Smolen, Baxter 102 and Byrne (e.g. Smolen et al., 1999, 2001, 2002). Of 103 particular interest here is Smolen et al. (1999) where the 104 relation between delays and macromolecular transport 105 was discussed. Specifically, their GRN model used a 106 delay to account for active transport of molecules and 107 showed that while such a model leads to oscillatory 108 behaviour, incorporating molecular diffusion supressed 109 oscillations. Other more recent models, including 110 models of the Hes1 system, the p53-Mdm2 system 111 and the NF- $\kappa$ B system, also showed that delays were 112 found to provoke oscillatory behaviour (Tiana et al., 113 2002; Jensen et al., 2003; Lewis, 2003; Monk, 2003; 114 Bernard et al., 2006). Theoretical models of synthetic 115 GRNs (e.g. repressilators) have also been proposed and 116 studied (Purcell et al., 2010; O'Brien et al., 2012), while 117 interest in modelling bacterial operons by Mackey and 118 co-workers (Yildirim and Mackey, 2003; Hilbert et al., 119 2011; Mackey et al., 2015) has added additional insight. 120

Early spatial models of theoretical intracellular systems were pioneered in the 1970s by Glass and ${ }_{123}$ co-workers (Glass and Kauffman, 1970; Shymko and 124 Glass, 1974) and again in the 1980s by Mahaffy and 125 co-workers (Busenberg and Mahaffy, 1985; Mahaffy, 126 1988; Mahaffy and Pao, 1984), where the focus was 127 on analysing generic systems with one-dimensional models. ODE models were reconfigured to incorporate a spatial dimension using reaction-diffusion PDEs and steady states and stability were determined with partic- ${ }_{129}$ ular attention paid to the geometry of the model. They ${ }_{130}$ coined the term "spatial switching" to indicate how 131 the system geometry can lead to different dynamical 132 behaviour. This approach has recently been extended 133 by Naqib et al. (2012). Other spatial models have ${ }_{134}$ focussed on the idea of modelling a cell using two (or ${ }_{135}$ more) compartments, to account for different processes 136 which occur in the nucleus and cytoplasm (see, for example, Sturrock et al., 2011, 2012); certain models incorporate both compartments and delays (e.g. Momiji and Monk, 2008). A two-dimensional spatial model of molecular transport inside a cell was formulated by Cangiani and Natalini (2010) and this general approach was adopted by Sturrock et al. (2011) to formulate and study a spatio-temporal model of the Hes1 GRN considering diffusion of the protein and mRNA. This model was then later extended to account for transport across the nuclear membrane and directed transport via microtubules (Sturrock et al., 2012). Other papers adopting an explicitly spatial approach include those of Szymańska et al. (2014), focussing on the role of transport via the microtubules, and Clairambault and co-workers (Dimitrio et al., 2013; Eliaš and Clairambault, 2014; Eliaš et al., 2014a,b), focussing on the p53 system.

In this paper we focus on the spatial component, by supposing that the different processes within a given GRN occur at specific sites. This approach removes the requirement to consider compartments and instead localises mRNA and protein production. The initial Hes 1 model is an extension of the model of Sturrock et al. $(2011,2012)$ and is inspired by the recent result of Chaplain et al. (2015) where it was proved rigorously that molecular diffusion causes oscillations. We develop and analyse spatio-temporal mathematical models for synthetic GRNs, focussing on the role of diffusion and the spatial location of the gene sites and protein production sites in generating and controlling the oscillatory dynamics.

The paper is structured as follows. In Section 2 we discuss the results of the canonical GRN, the Hes1 system. In Sections 3 and 4 we develop models and present simulation results for three different synthetic GRNs, specifically repressilators and activatorrepressors. Discussions, conclusions and directions for future work in this area are given in the final Section 5.

\section{The Hes1 System}

The Hes1 protein is a member of the family of basic helix-loop-helix (bHLH) transcription factors and is known to repress the transcription of its own gene through direct binding to regulatory sequences in the Hes 1 promoter (Hirata et al., 2002). For this reason, it may be termed the canonical transcription factor or canonical gene regulatory network. It is known that periodically changing levels of Hes 1 protein controls 
embryonic development, specifically in correctly timed somite segmentation (see, for example, Kageyama et al., 2007). Mathematical modelling is particularly well suited to the relatively simple Hes1 system, which is controlled by way of a single negative feedback loop between its mRNA and protein. Of particular interest here is spatial modelling. Sturrock et al. (2011) showed that a two compartment, nucleus-cytoplasm reactiondiffusion model gives rise to oscillatory behaviour, while Chaplain et al. (2015) rigorously proved that the diffusion parameter controls whether or not the system oscillates. Here we modify the model used by Chaplain et al. (2015) to incorporate sites at which the hes1 mRNA and Hes1 protein will be produced. For the purposes of discussion we will refer to locations of mRNA production as "gene-sites" and protein production as "production sites". We present results for a 1D interval model, however they equally apply to models of the system in other geometries, specifically 2D circular and elliptical and 3D spherical, (more details can be found in Appendix B). We consider the non-dimensional (see 2.1 for details of the non-dimensionalisation) form of the $1 \mathrm{D}$ model to be:

$$
\begin{array}{r}
\frac{\partial m}{\partial t}=D \frac{\partial^{2} m}{\partial x^{2}}+\frac{\alpha_{m}}{1+p^{h}} \delta_{x_{m}}^{\varepsilon}(x)-\mu m \\
\frac{\partial p}{\partial t}=D \frac{\partial^{2} p}{\partial x^{2}}+\alpha_{p} m \delta_{x_{p}}^{\varepsilon}(x)-\mu p
\end{array}
$$

where $m(x, t)$ and $p(x, t)$ are the concentrations of hes 1 mRNA and Hes1 protein, respectively. Initially (and for ${ }^{187}$ simplicity) we assume that both mRNA and protein dif- 188 fuse through the cell with the same constant diffusion 189 coefficient, $D$, and are subject to degradation (propor- 190 tional to their concentrations) at the same rate, $\mu$. The 191 protein is translated at a production site located at po- 192 sition $x_{p}$, at a rate $\alpha_{p}$ and proportional to the level of 193 mRNA. The presence of the protein then represses the 194 production of mRNA (modelled by a Hill function with 195 Hill coefficient $h$ ) which undergoes transcription at a 196 gene-site located at position $x_{m}$, at a rate $\alpha_{m}$. As such 197 the Hes 1 system consists of a simple negative feedback 198 loop (see Figure 1 for a simple schematic of the system). ${ }^{199}$

Following Chaplain et al. (2015) we use a Dirac approx- ${ }^{201}$ imation of the $\delta$-distribution function located at the gene ${ }^{202}$ and protein production sites $x_{i}$, where $i=\{m, p\}$, such ${ }^{203}$ that

$$
\delta_{x_{i}}^{\varepsilon}(x)= \begin{cases}\frac{1}{2 \varepsilon}\left[1+\cos \left(\frac{\pi\left(x-x_{i}\right)}{\varepsilon}\right)\right] & \left|x-x_{i}\right|<\varepsilon, \\ 0 & \left|x-x_{i}\right| \geq \varepsilon\end{cases}
$$

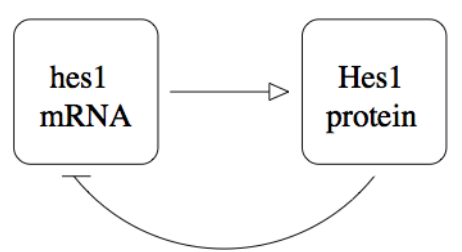

Figure 1: Simple schematic of the Hes1 gene regulatory system. Hes 1 protein is produced from hes 1 mRNA via translation, but then inhibits the production of hes1 mRNA (represses or down-regulates transcription).

with $\varepsilon>0$ a small parameter indicating the half-width of the function. We consider a symmetric 1D interval $x \in[-1,1]$, and the positions of the gene and protein production sites will be varied. We assume zero-flux (Neumann) boundary conditions on the edges of the domain such that:

$$
\begin{aligned}
& \frac{\partial m(-1, t)}{\partial x}=\frac{\partial m(1, t)}{\partial x}=0, \\
& \frac{\partial p(-1, t)}{\partial x}=\frac{\partial p(1, t)}{\partial x}=0,
\end{aligned}
$$

185

and initial conditions, i.e.,

$$
m(x, 0)=0, \quad p(x, 0)=0 .
$$

\subsection{Model Parameters}

The non-dimensional parameters, used in our simulations are given in Table 1. These parameters are taken from Sturrock et al. (2012), where a detailed discussion of parameter choices is provided. They carried out a full parameter analysis to determine the ranges for each parameter for which oscillations were observed as well as indicating how these values correspond with results from the experimental literature. Our initial assumption is that diffusion is a constant and the other parameters are fixed. Sturrock et al. (2012) found that changes to the parameters lead to changes in the nature of oscillations, for example, changes to the period. It is realistic to expect that changes to the intracellular (and extracellular) environment would affect these parameters. For example, changes to the transcription/translation machinery due to temperature may affect the rates of mRNA and protein production. Such changes would then affect the period of oscillation

In non-dimensionalising the original Hes1 system (see Sturrock et al. (2012) for more details) the relationships between the non-dimensional parameters $a$ and their di- 


\begin{tabular}{|l|l|c|}
\hline Parameter & Description & Value \\
\hline$D$ & diffusion coefficient & 0.00075 \\
$\alpha_{m}$ & mRNA transcription rate & 1.0 \\
$\alpha_{p}$ & protein translation rate & 2.0 \\
$\mu$ & natural degradation rate & 0.03 \\
$h$ & hill coefficient & 5 \\
$\varepsilon$ & small parameter used in & 0.01 \\
& delta-function approx. & \\
\hline
\end{tabular}

Table 1: Non-dimensional parameter values used throughout our simulations. Note initially we consider that both mRNA and protein diffuse at the same rate such that $D_{m}=D_{p}=D$.

mensional counterparts $[a]$ are given as follows:

$$
\begin{gathered}
D=\frac{\tau[D]}{L^{2}}, \quad \alpha_{m}=\frac{\tau\left[\alpha_{m}\right]}{m_{0}}, \\
\alpha_{p}=\frac{\tau m_{0}\left[\alpha_{p}\right]}{p_{0}}, \quad \mu=\tau[\mu],
\end{gathered}
$$

where $\tau$ and $L$ are a reference time and length, respectively and $m_{0}$ and $p_{0}$ are reference concentrations of mRNA, $m$ and protein, $p$. Following Sturrock et al. (2012) we take $m_{0}=0.0015 \mu \mathrm{M}$ and $p_{0}=0.001 \mu \mathrm{M}$. Taking their lead we suppose that a cell is of width $30 \mu \mathrm{m}$, and as such $L=15 \mu \mathrm{m}$ (since our domain has length $2 L$ ). Equally by comparing the periods of oscillation we observe (for the Hes1 system) with experimental data (which found the period of oscillations to be 2 hours, Hirata et al., 2002) we take $\tau=22.5$ (since oscillations occur with a period of approximately 320 time units). Using these reference values the dimensional parameters can be determined and are given in Table 2. We note that the diffusion coefficient lies within the range of diffusion coefficients noted by Oeffinger and Zenklusen (2012) (these varied from $0.005 \mu \mathrm{m}^{2} / \mathrm{s}$ up to more than $1 \mu^{2} / \mathrm{s}$ ). While most of the parameters in our model are not precisely determined experimentally, we note that we find the behaviour reported for ranges of values for each parameter (cf. Sturrock et al. (2012))

\begin{tabular}{|c|l|c|}
\hline Parameter & Dimensions & Value \\
\hline$[D]$ & $\mu \mathrm{m}^{2} \mathrm{~s}^{-1}$ & $7.5 \times 10^{-3}$ \\
{$\left[\alpha_{m}\right]$} & $\mathrm{Ms}^{-1}$ & $6.67 \times 10^{-11}$ \\
{$\left[\alpha_{p}\right]$} & $\mathrm{s}^{-1}$ & 0.0593 \\
{$[\mu]$} & $\mathrm{s}^{-1}$ & $1.33 \times 10^{-3}$ \\
\hline
\end{tabular}

Table 2: Dimensional parameter values.

\subsection{Varying Protein Production Site Position}

We solve system (1), subject to the boundary conditions (3) and initial conditions (4), first in Matlab using the inbuilt pdepe solver and then using COMSOL, with comparable results. In Figure 2 we display the time-variation of the total concentrations of mRNA (top panel) and protein (bottom panel). Since our interest lies in whether the separation between the mRNA and protein production affects whether the system will oscillate, we choose to fix the mRNA gene-site (at $x_{m}=0.0$ ) and vary the position of two protein production sites (symmetric about $x_{m}$ ). We observe that if the protein production sites are either too close to (solid cyan curves, $x_{p}= \pm 0.1$ ) or too far away from (red dashed curves, $x_{p}= \pm 0.9$ ) the mRNA gene-site, the system will not oscillate. Instead, in both cases, the system tends towards a constant level (low or high, respectively) of both mRNA and protein. For the case $x_{p}= \pm 0.9$, the system does initially exhibit oscillatory behaviour, however oscillations are quickly damped.

For protein production sites which are adequately separated from the mRNA gene-site (blue dashed, black solid and green dotted curves), oscillations occur. The periods we observe exhibit quite wide variation, with the period increasing in proportion to the distance between production sites. For example, the period varies from 200 time units (for $x_{p}= \pm 0.3$ ) to 360 time units (for $x_{p}= \pm 0.7$ ). The amplitude of mRNA oscillations increases as the protein production site moves further away, suggesting that an increase in separation leads to higher peak levels of mRNA. However, the highest peak level for the protein is observed for a mid-range separation distance, e.g. $x_{p}= \pm 0.5$. See Figure A.14 of Appendix A for the full space-time behaviour of mRNA and protein concentrations for the Hes1 system (1) with $x_{m}=0.0$ and $x_{p}=\{ \pm 0.1, \pm 0.5, \pm 0.9\}$.

Our results indicate that separation between mRNA and protein production sites can affect whether oscillations occur, even for an "optimum" diffusion rate. Moreover the precise distance can affect oscillation amplitudes and periods. This result demonstrates that it is important not to neglect the spatial aspect in modelling GRNs. For the purpose of discussion within this paper (for our initial diffusion coefficient regime) we will consider a separation distance of 0.5 length units as optimum, while separations of 0.1 and 0.9 length units are considered to be too short and too long, respectively. 

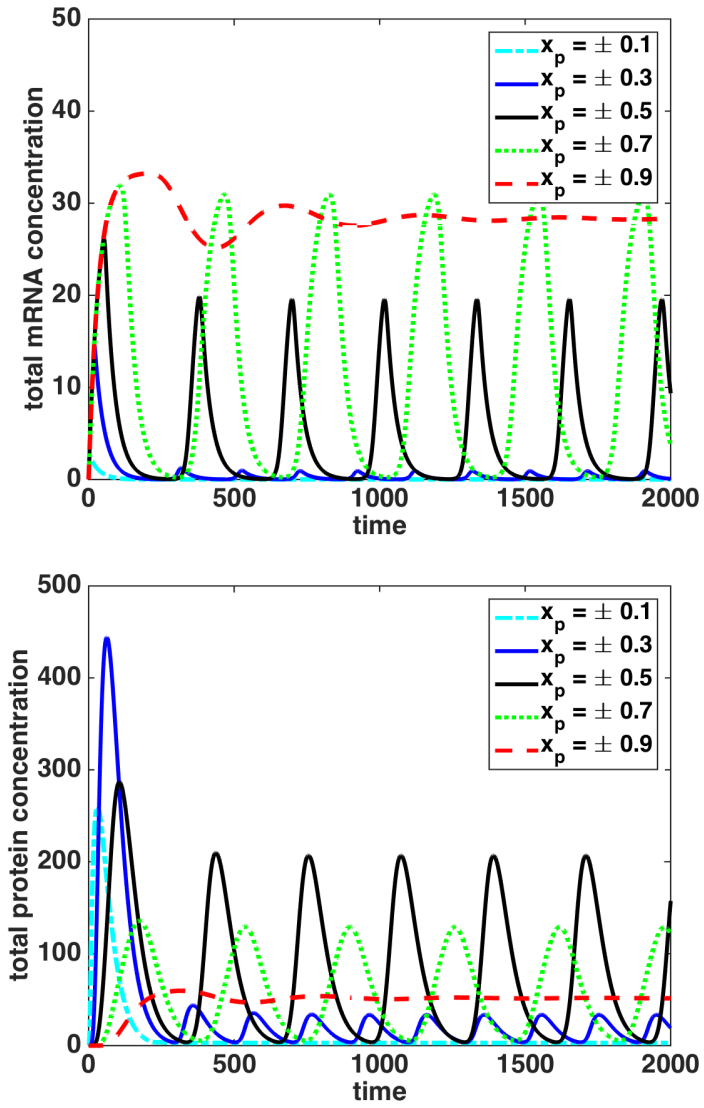

Figure 2: Total mRNA (top panel) and protein (bottom panel) concentrations, for the Hes1 system, (1). The mRNA gene-site is located at $x_{m}=0.0$ and the protein production sites are located at $x_{p}=$ $\{ \pm 0.1, \pm 0.3, \pm 0.5, \pm 0.7, \pm 0.9\}$ (see legend).

\subsection{Varying the Diffusion Coefficients}

We now consider the effect of varying the diffusion coefficients of mRNA and protein. Initial investigations show that oscillations can be obtained for a range of diffusion coefficients (as for Chaplain et al., 2015, etc) but moreover that the particular combination of diffusion coefficients along with position of production sites dictates whether oscillations occur, and the nature 297 of oscillations. If diffusion rates, for either mRNA or 298 protein (or both) are increased/decreased, a correspond- 299 ing increase/decrease in separation between production 300 sites may also be required to generate oscillations.

In Figure 3 we indicate four separate cases, all of 303 which lead to oscillations. In the first and second cases 304 $x_{m}=0.0$ and $x_{p}= \pm 0.9$, creating a separation between 305 production sites which did not lead to oscillations for 306
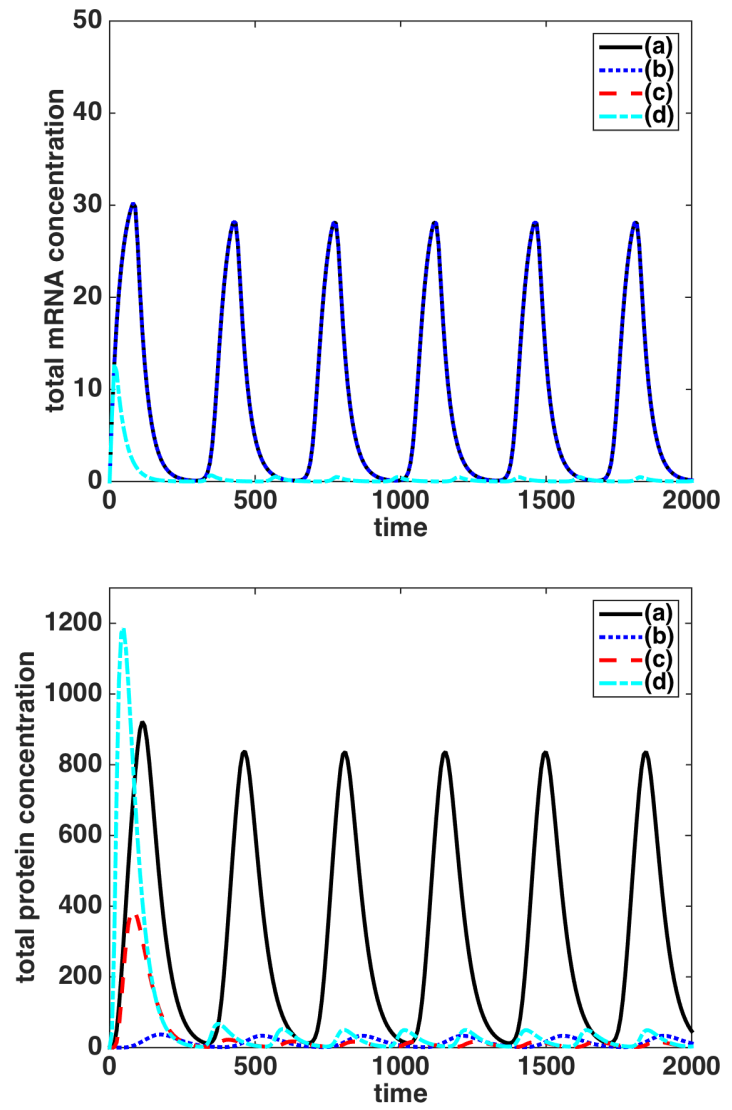

Figure 3: Total mRNA (top panel) and protein (bottom panel) concentrations, for the Hes1 system (1). Case (a) - solid black curve: $D_{m}=7.5 \times 10^{-3}, D_{p}=7.5 \times 10^{-4}$, $x_{m}=0.0$ and $x_{p}= \pm 0.9$. Case (b) - blue dotted curve: $D_{m}=7.5 \times 10^{-4}, D_{p}=7.5 \times 10^{-3}, x_{m}=0.0$ and $x_{p}=$ \pm 0.9 . Case (c) - red dashed curve: $D_{m}=7.5 \times 10^{-5}$, $D_{p}=7.5 \times 10^{-4}, x_{m}=0.0$ and $x_{p}= \pm 0.15$. Case (d) - cyan dot-dashed curve: $D_{m}=7.5 \times 10^{-4}, D_{p}=$ $7.5 \times 10^{-5}, x_{m}=0.0$ and $x_{p}= \pm 0.15$. Note that in the top panel (for the mRNA concentration) the solid black curve lies underneath the blue dotted curve and the red dashed curve lies underneath the cyan dot dashed curve.

our original diffusion coefficient regime (where the diffusion coefficients are both equal to $D$, as specified in Table 1). By increasing either the diffusion of mRNA, in case (a), or protein, in case (b), by one order of magnitude, we show that oscillations in the system are now possible. In the third and fourth cases $x_{m}=0.0$ and $x_{p}= \pm 0.15$, again creating a separation between production sites which did not lead to oscillations for the original diffusion coefficient regime. By decreasing either the diffusion of mRNA, in case (c), or protein, 
in case (d), by one order of magnitude, we show that ${ }_{357}$ oscillations in the system are now possible. Note, even ${ }_{358}$ when we reduce the diffusion rate of either mRNA or 359 protein by one order of magnitude, a separation of 0.1360 remains too close to achieve oscillations. However, by ${ }_{361}$ reducing both diffusion rates we can obtain oscillations 362 at this separation. Thus, for oscillations to occur ${ }_{363}$ either the diffusion coefficients or gene-site separation 364 distances (or both) should be optimised.

Since mRNA molecules are smaller than protein molecules, one might infer that mRNA diffuses faster than protein (cf. Stokes-Einstein Law, Miller, 1924). For the remainder of this paper we will discuss results for two diffusion coefficient regimes. In the first both mRNA and protein diffuse at a rate $D$. In the second mRNA diffuses more quickly at a rate $D_{m}=0.0075$ while diffusion of protein remains at a rate $D_{p}=D$. All other parameters remain as in Table 1. For this second diffusion coefficient regime we note that oscillations are now possible at a separation of 0.9 length units (and are still not found for a separation of 0.1 length units). See Figure A.15 of Appendix A for the full space-time behaviour of mRNA and protein concentrations for the Hes1 system (1) with $x_{m}=0.0$ and $x_{p}=\{ \pm 0.1, \pm 0.5, \pm 0.9\}$, for this second diffusion coefficient regime.

We repeated the investigation into the Hes1 system for different geometries considering a circular, elliptical and spherical domain (see Appendix B). The results were qualitatively comparable, confirming that both the size of the diffusion coefficients and the distance between production sites (or zones) must be optimised for the Hes1 system to oscillate. We note that some alterations to parameters may be required as the dimensions of the domain are increased. Armed with our results for the Hes1 system we consider the role of production site position in multi-gene GRNs. In particular we shall study the mechanics of two types of synthetic system. The first type consists of down-regulation alone, where a given gene in the cycle represses the next, which we will refer to as a repressilator. The second type will combine both up-regulation/activation and down-regulation/repression of genes. We will refer to such systems as activator-repressor systems.

\section{Synthetic GRNs: Repressilators}

The term repressilator (first coined by Elowitz and Leibler, 2000) has been reserved for a system of three genes which couple to form a cycle of negative feedback loops. We choose to use this terminology, for ease of reference, for any n-gene system for which the protein of any given gene inhibits the production of the mRNA for the subsequent gene. Under this terminology, the Hes1 system can be termed a one-gene repressilator and we base our synthetic multi-gene repressilator on the Hes1 system structure. As such the equations in 1D are taken to be:

$$
\begin{gathered}
\frac{\partial m_{i}}{\partial t}=D_{m} \frac{\partial^{2} m_{i}}{\partial x^{2}}+\frac{\alpha_{m}}{1+p_{j}^{h}} \delta_{x_{m i}}^{\varepsilon}(x)-\mu_{m} m_{i}, \\
\frac{\partial p_{i}}{\partial t}=D_{p} \frac{\partial^{2} p_{i}}{\partial x^{2}}+\alpha_{p} m_{i} \delta_{x_{p i}}^{\varepsilon}(x)-\mu_{p} p_{i},
\end{gathered}
$$

where $i=\{1,2,3, \ldots n\}$ and, since the repression of mRNA comes from the preceding protein in the system, $j=\{n, 1,2,3, \ldots(n-1)\}$, for an $n$-gene system. As before we use a Dirac approximation of the $\delta$-distribution function, this time located at the production sites $x_{m i}$ and $x_{p i}$, with $i$ as above. In our simulations we consider the effect of varying the position of these production sites. The boundary conditions and initial conditions are, as before, such that:

$$
\begin{aligned}
\frac{\partial m_{i}(-1, t)}{\partial x}=\frac{\partial m_{i}(1, t)}{\partial x} & =0, \\
\frac{\partial p_{i}(-1, t)}{\partial x}=\frac{\partial p_{i}(1, t)}{\partial x} & =0, \\
m_{i}(x, 0)=0 & p_{i}(x, 0)=0 .
\end{aligned}
$$

\subsection{Two-gene Repressilator}

We begin our analysis of multi-gene repressilators by considering a two-gene (or species) system. A simple schematic of a generic two-gene repressilator is shown in Figure 4.

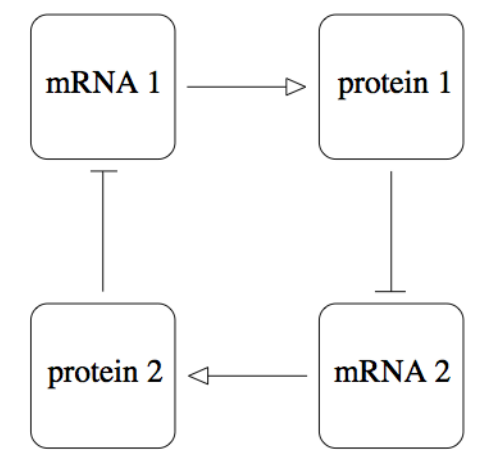

Figure 4: Simple schematic of the two-gene repressilator system. Each species mRNA produces its own protein. Each species' protein inhibits the production of the other species' mRNA. 
We solve the $1 \mathrm{D}$ system (6), where $n=2$, with boundary conditions (7) and initial conditions (8) using the pdepe solver in Matlab (comparable results were obtained using COMSOL). Parameters remain as for the Hes1 system and are given in Table 1. By solving the system for numerous production site scenarios and varying the diffusion coefficients, we find that the two-gene repressilator system is a "weak" oscillator, in that it only oscillates for a very limited set of conditions. In Figure 5 we provide the results for four key cases, (a)-(d), under both diffusion coefficient regimes. Since we focus our attention solely on distinguishing between oscillating and non-oscillating cases, we choose not to report on the behaviour of the system for the cases which do not show periodic behaviour. In general, we note that alternative scenarios may result in persistent high or low concentrations of the mRNAs and proteins. To that end we graph the concentrations of species 1 mRNA only (all other concentrations behave qualitatively in the same manner).

In case (a) both genes have the same production sites which are optimally separated for both diffusion coefficient regimes $\left(x_{m 1}=x_{m 2}=0.0\right.$ and $\left.x_{p 1}=x_{p 2}= \pm 0.5\right)$. We observe oscillations, for both regimes, which match closely with the oscillations for the one-gene Hes1 system. In this case, as we might expect, the two-gene system acts just as if it were a single-gene system. Nothing in the equations or numerical code distinguishes species 1 from species 2. In case (b) the two genes have different production sites although they remain optimally separated for both diffusion coefficient regimes $\left(x_{m 1}=\right.$ $\pm 0.2, x_{m 2}= \pm 0.4, x_{p 1}= \pm 0.7$ and $x_{p 2}= \pm 0.5$ ). Oscillations are not observed in either regime. In case (c) both genes have the same production sites with separation distances only optimal for the second diffusion coefficient regime $\left(x_{m 1}=x_{m 2}=0.0\right.$ and $\left.x_{p 1}=x_{p 2}= \pm 0.9\right)$. Oscillations are only seen for the second diffusion coefficient regime. In case (d) the two genes have different production sites with separation distances remaining optimal for only the second diffusion coefficient regime $\left(x_{m 1}=0.0, x_{m 2}= \pm 0.1, x_{p 1}= \pm 0.9\right.$ and $\left.x_{p 2}= \pm 1.0\right)$. In this case neither diffusion coefficient regime leads to ${ }^{432}$ oscillations. Our investigations indicate that if the pro- ${ }^{433}$ duction sites for the two genes are different then oscillations will not occur, regardless of whether gene-site position and diffusion coefficients are optimum or not. ${ }^{435}$ Oscillations may only be obtained if the two genes share ${ }_{436}$ the same production sites, when the system effectively ${ }_{437}$ behaves like a one-gene repressilator, the Hes1 system. ${ }_{438}$ However, in such a case, the separation between pro- ${ }_{439}$
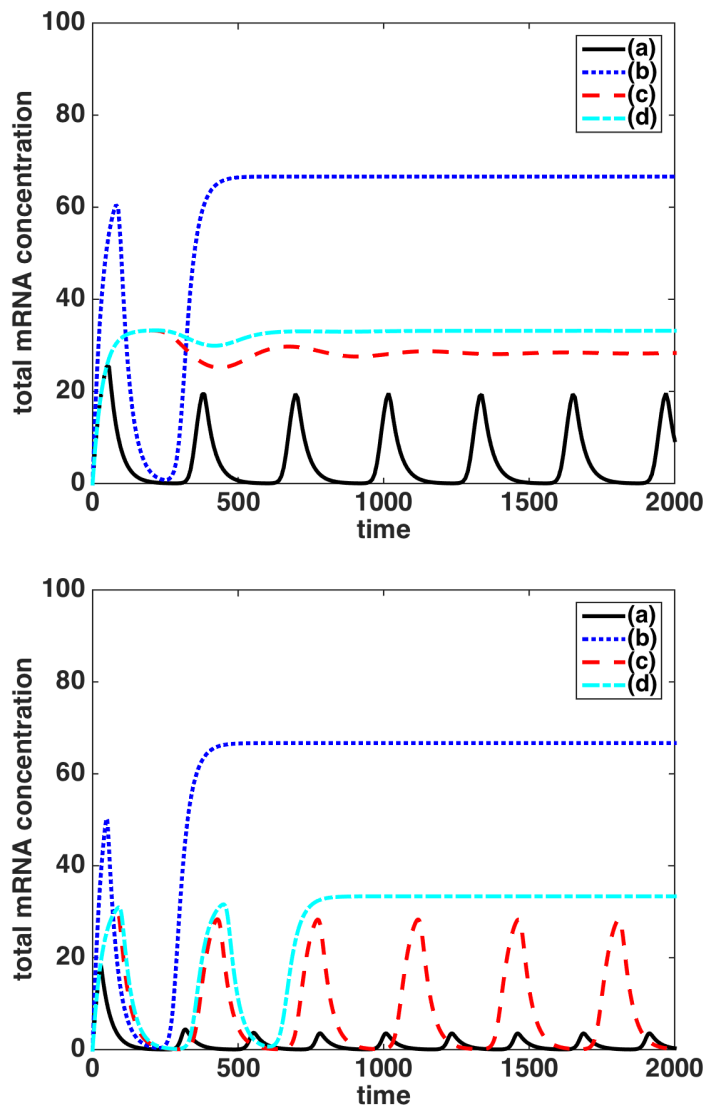

Figure 5: Total mRNA concentrations for species 1 for the two-gene repressilator under both diffusion coefficient regimes. Top panel: first regime, $D_{m}=D_{p}=D$. Bottom panel: second regime, $D_{m}=0.0075$ and $D_{p}=$ $D$. Case (a) - solid black curve: the production sites are $x_{m 1}=x_{m 2}=0.0$ and $x_{p 1}=x_{p 2}= \pm 0.5$. Case (b) blue dotted curve: the production sites are $x_{m 1}= \pm 0.2$, $x_{m 2}= \pm 0.4, x_{p 1}= \pm 0.7$ and $x_{p 2}= \pm 0.9$. Case (c) - red dashed curve: the production sites are $x_{m 1}=x_{m 2}=0.0$, $x_{p 1}=x_{p 2}= \pm 0.9$. Case (d) - cyan dot-dashed curve: the production sites are $x_{m 1}=0.0, x_{m 2}= \pm 0.1, x_{p 1}= \pm 0.9$ and $x_{p 2}= \pm 1.0$.

duction sites must then be optimised in relation to the diffusion coefficients in order to obtain oscillations. In support of these results we note that we have obtained comparable results for other geometries.

\subsection{Three-gene Repressilator}

We now consider the behaviour of a three-gene system, by solving system (6), where $n=3$, with boundary conditions (7) and initial conditions (8), as previously, using the pdepe solver in Matlab (comparable results 
were obtained using COMSOL). Our investigation 449 indicates that the three-gene repressilator oscillates 450 more readily.
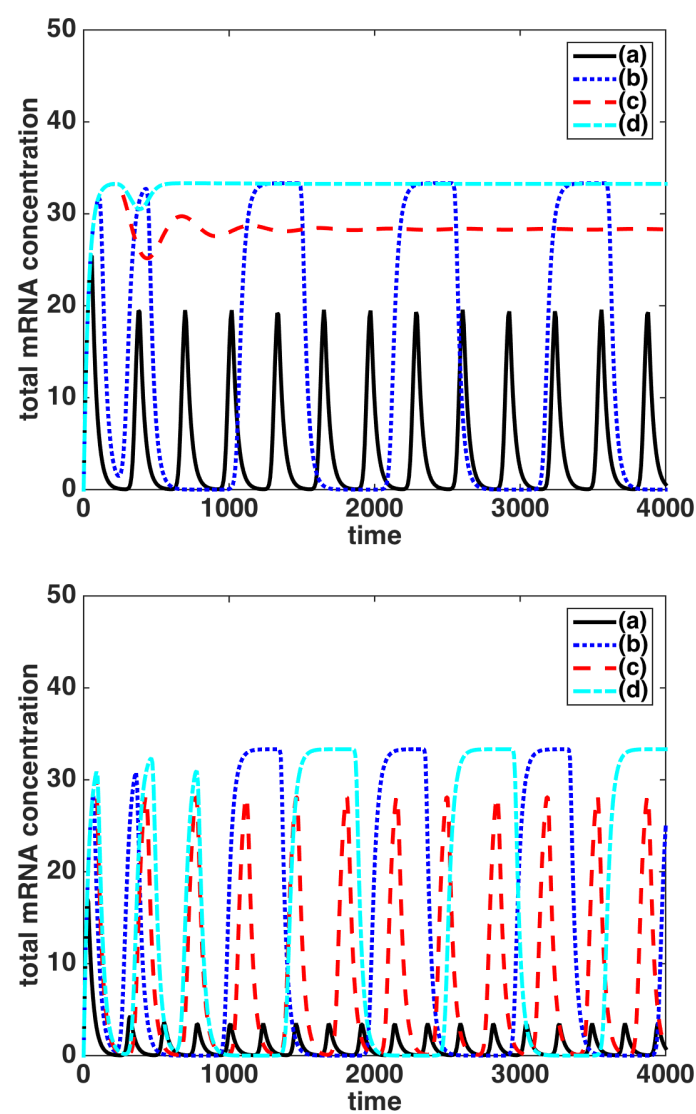

Figure 6: Total mRNA concentrations for species 1 for the three-gene repressilator under both diffusion coefficient regimes. Top panel: first regime, $D_{m}=D_{p}=D$. Bottom panel: second regime, $D_{m}=0.0075$ and $D_{p}=$ $D$. Case (a) - solid black curve: the production sites are $x_{m 1}=x_{m 2}=x_{m 3}=0.0$ and $x_{p 1}=x_{p 2}=x_{p 3}= \pm 0.5$. Case (b) - blue dotted curve: the production sites are $x_{m 1}=0.0, x_{m 2}= \pm 0.2, x_{m 2}= \pm 0.4, x_{p 1}= \pm 0.5$, $x_{p 2}= \pm 0.7$ and $x_{p 3}= \pm 0.9$. Case (c) - red dashed curve: the production sites are $x_{m 1}=x_{m 2}=x_{m 3}=0.0$ and $x_{p 1}=x_{p 2}=x_{p 3}= \pm 0.9$. Case (d) - cyan dot-dashed curve: the production sites are $x_{m 1}=0.0, x_{m 2}= \pm 0.05$, $x_{m 3}= \pm 0.1, x_{p 1}= \pm 0.9, x_{m 2}= \pm 0.95$ and $x_{p 3}= \pm 1.0$.

In Figure 6 we show the concentrations of species 1496 mRNA (since all other concentrations behave qualitat- 497 ively the same), and compare to the two-gene system 498 by considering comparable cases. In case (a) all three 499 genes have the same production sites which are op- 500 timally separated for both diffusion coefficient regimes $\left(x_{m 1}=x_{m 2}=x_{m 3}=0.0\right.$ and $\left.x_{p 1}=x_{p 2}=x_{p 3}= \pm 0.5\right)$. We note oscillations (for both regimes) across all species. The full space-time behaviour of all three mRNAs and proteins for this case are given in Figure A.16 of Appendix A. We observe that the system behaves as if there were three copies of the Hes1 gene.

In case (b) all three genes have different production sites but remain optimally separated for both diffusion coefficient regimes $\left(x_{m 1}=0.0, x_{m 2}= \pm 0.2\right.$, $x_{m 2}= \pm 0.4, x_{p 1}= \pm 0.5, x_{p 2}= \pm 0.7$ and $\left.x_{p 3}= \pm 0.9\right)$. In this case, unlike for the two-gene repressilator, oscillations are observed (for both regimes). We note that both the period and amplitude of the oscillations for all three mRNAs and proteins are greater compared to case (a). Although two of the mRNAs are now produced in two locations rather than one, effectively doubling production, this cannot fully account for the differences. The full space-time behaviour of all three mRNAs and proteins for this case are given in Figure A.17 of Appendix A. We observe that the system exhibits longer periods, with an increase in both the time between consecutive peaks and time at peak amplitude.

In case (c) all three species have the same production sites with all separation distances optimal for only the second diffusion coefficient regime $\left(x_{m 1}=x_{m 2}=x_{m 3}=0.0\right.$ and $\left.x_{p 1}=x_{p 2}=x_{p 3}= \pm 0.9\right)$. In case (d) all three species have different production sites and all separation distances are only optimal the second diffusion coefficient regime $\left(x_{m 1}=0.0\right.$, $x_{m 2}= \pm 0.05, x_{m 3}= \pm 0.1, x_{p 1}= \pm 0.9, x_{m 2}= \pm 0.95$ and $x_{p 3}= \pm 1.0$ ). In these cases oscillations are only observed for the second diffusion coefficient regime. However, since oscillations are observed for both case (c) and (d) this, again, indicates that the three-gene repressilator does not require the production sites to be in the same location to oscillate. Again we note that the period of oscillations (when they occur) for all three species is greater in case (d) compared to case (c). This suggests that when the production sites are in different locations the period is increased, i.e. it takes longer to cycle through the system.

Since we find that the three-gene repressilator will oscillate when each of the three genes are produced in different locations we can extend our investigation. We consider a wide range of scenarios for production site position and find that the three-gene repressilator continues to oscillate readily. We investigate the 
difference in dynamics when the separation distance 524 from and on one or two mRNA(s) or protein(s) are 525 non-optimum i.e. too far apart or too close.

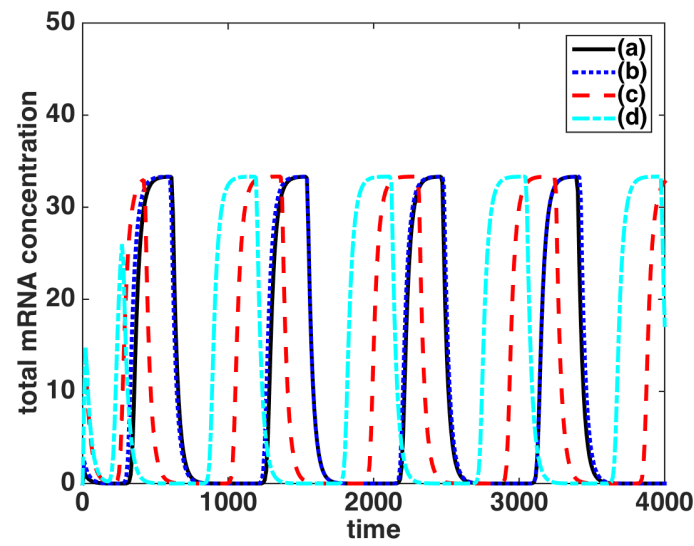

Figure 7: Total mRNA concentration for species 1 over time for the three-gene repressilator, under the first diffusion coefficient regime, $D_{m}=D_{p}=D$. Case (a) solid black curve: the production sites are $x_{m 1}=x_{m 2}=$ $x_{m 3}=0.0, x_{p 1}=x_{p 2}= \pm 0.5$ and $x_{p 3}= \pm 0.1$. Case (b) - blue dotted curve: the production sites are $x_{m 1}=$ $x_{m 2}=x_{m 3}=0.0, x_{p 1}= \pm 0.5$ and $x_{p 2}=x_{p 3}= \pm 0.1$ - Case (c) - red dashed curve: the production sites are $x_{m 1}=x_{m 2}=0.0, x_{m 3}= \pm 0.4$ and $x_{p 1}=x_{p 2}=$ $x_{p 3}= \pm 0.5$. Case $(\mathrm{d})$ - cyan dot-dashed curve: the production sites are $x_{m 1}=0.0, x_{m 2}=x_{m 3}= \pm 0.4$ and $x_{p 1}=x_{p 2}=x_{p 3}= \pm 0.5$.

We find that the three-gene repressilator will oscillate when the separation distances to and from one or two mRNA(s) or protein(s) are too small, providing at least one separation pair remains optimum. In Figure 7 we show the oscillatory behaviour of species 1 mRNA under the first diffusion coefficient regime for four specific scenarios (although a much more comprehensive set have been investigated). In case (a) the production sites are $x_{m 1}=x_{m 2}=x_{m 3}=0.0$, $x_{p 1}=x_{p 2}= \pm 0.5$ and $x_{p 3}= \pm 0.1$ (solid black curve), i.e. one pair of separation distances, acting on and from species 3 protein are too small. In case (b) the production sites are $x_{m 1}=x_{m 2}=x_{m 3}=0.0, x_{p 1}= \pm 0.5$ and $x_{p 2}=x_{p 3}= \pm 0.1$ (blue dotted curve), i.e. two 542 pairs of separation distances, acting on and from 543 species 2 and 3 proteins are too small. In case (c) the 544 production sites are $x_{m 1}=x_{m 2}=0.0, x_{m 3}= \pm 0.4545$ and $x_{p 1}=x_{p 2}=x_{p 3}= \pm 0.5$ (red dashed curve), 546 i.e. one pair of separation distances, acting on and 547 from species 3 mRNA are too small. In case (d) the production sites are $x_{m 1}=0.0, x_{m 2}=x_{m 3}= \pm 0.4$ and $x_{p 1}=x_{p 2}=x_{p 3}= \pm 0.5$ (cyan dot-dashed curve), i.e. two pairs of separation distances, acting on and from species 2 and 3 mRNAs are too small. In all four cases we observe comparable oscillations with similar amplitude and period. These results were found regardless of which species was picked to have optimum geneand protein production site separation. This suggests that whether the system will oscillate or not is governed by the greatest separation distance, rather than the least. Provided that this greatest distance is within the optimum range, the system will oscillate. Again we note that the amplitude and period of the oscillations we observe are higher than for the case when all three species share the same mRNA and protein production sites.

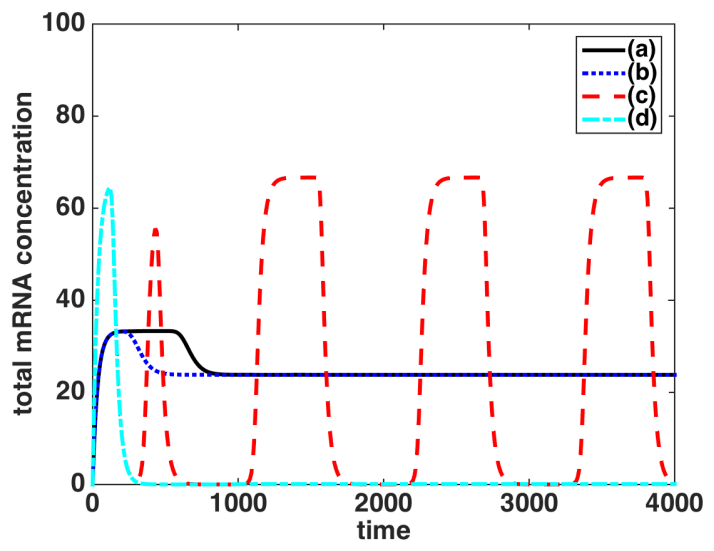

Figure 8: Total mRNA concentration for species 1 for the three-gene repressilator, under the first diffusion coefficient regime, $D_{m}=D_{p}=D$. Case (a) - solid black curve: the production sites are $x_{m 1}=x_{m 2}=x_{m 3}=0.0$, $x_{p 1}=x_{p 2}= \pm 0.5$ and $x_{p 3}= \pm 0.9$. Case (b) - blue dotted curve: the production sites are $x_{m 1}=x_{m 2}=x_{m 3}=0.0$, $x_{p 1}= \pm 0.5$ and $x_{p 2}=x_{p 3}= \pm 0.9$. Case (c) - red dashed curve: the production sites are $x_{m 1}=x_{m 2}= \pm 0.4$, $x_{m 3}=0.0, x_{p 1}=x_{p 2}=x_{p 3}= \pm 0.9$. Case (d) - cyan dot-dashed curve: the production sites are $x_{m 1}= \pm 0.4$, $x_{m 2}=x_{m 3}=0.0, x_{p 1}=x_{p 2}=x_{p 3}= \pm 0.9$.

Conducting a similar investigation considering cases when the separation distances to and from one or two mRNA(s) or protein(s) are too large, we find that the system does not necessarily oscillate. This would confirm our assertion that the system oscillates only when the greatest separation distance is optimised. In 
Figure 8 we show the behaviour of species 1 mRNA 599 under the first diffusion coefficient regime for four 600 specific scenarios. In case (a) the production sites 601 are $x_{m 1}=x_{m 2}=x_{m 3}=0.0, x_{p 1}=x_{p 2}= \pm 0.5602$ and $x_{p 3}= \pm 0.9$ (solid black curve), i.e. one pair of 603 separation distances, acting on and from species 3604 protein are too great. In case (b) the production sites are $x_{m 1}=x_{m 2}=x_{m 3}=0.0, x_{p 1}= \pm 0.5$ and $x_{p 2}=x_{p 3}= \pm 0.9$ (blue dotted curve), i.e. two pairs of separation distances, acting on and from species 2 and 3 proteins are too great. In case (c) the production sites are $x_{m 1}=x_{m 2}= \pm 0.4, x_{m 3}=0.0$ and $x_{p 1}=x_{p 2}=x_{p 3}= \pm 0.9$ (red dashed curve), i.e. one pair of separation distances, acting on and from species 3 mRNA are too great. In case (d) the production sites are $x_{m 1}= \pm 0.4, x_{m 2}=x_{m 3}=0.0$ and $x_{p 1}=x_{p 2}=x_{p 3}= \pm 0.9$ (cyan dot-dashed curve), i.e. two pairs of separation distances, acting on and from species 2 and 3 mRNAs are too great. Only case (c) oscillates.

All of our findings together suggest that the threegene repressilator system is a more robust oscillator than the two-gene repressilator. It will oscillate when species do not share production sites and requires only one separation distance to be optimal, other distances can be too close (but not too far) and the system will still oscillate. We have found comparable results in other geometries.

\section{3. n-gene Repressilators and Summary}

In order to make comments about potential $n$-gene repressilators, we consider four-, five-, six- and sevengene systems (Figures not provided). We observe that the behaviour of the four-gene is similar to that of the two-gene repressilator (preferentially oscillating when the production sites for the four genes are in the same location). On the other hand, and much like the threegene repressilator, the five-gene repressilator will oscillate for a range of conditions when the production sites are different. This suggests that the three-(and five)gene repressilators are more robust than the two-(and four-)gene repressilators. For six and seven-gene systems, we find oscillations for a wider range of cases, although the six-gene system oscillates less frequently than the seven-gene system. Increasing the number of genes in a repressilator system makes the system more robust and more likely to oscillate, with a bias towards an odd number of genes which (for the cases we con- ${ }^{636}$ sider) are more robust than the even number cases. In ${ }^{637}$ particular, for the cases studied here, repressilator sys- 638 tems with distinct production sites preferentially oscil- 639 late for systems with an odd number of genes. Since it is highly likely that the production sites of different genes are at different locations, this is an important result. If oscillations are to be achieved the separation distances between production sites of mRNA and protein must be optimised in relation to the rate of diffusion.

\section{Synthetic GRNs: Activator-Repressor Systems}

In this section we consider two different cases which we broadly classify as activator-repressor systems. For each we base our model on the repressilator system but with a change to the production term of one (or more) mRNA(s) so that it is promoted (rather than repressed) by the presence of the "preceding" protein in the chain. The terms we consider are

$$
\frac{\alpha_{m} p_{j}^{h}}{1+p_{j}^{h}},
$$

and

$$
\alpha_{m}+\frac{\beta_{m} p_{j}^{h}}{1+p_{j}^{h}},
$$

respectively. In case (A) the rate of production of an mRNA increases in proportion to the amount of the preceding protein (although this rate is capped and as such the actual rate is always less than the baseline rate of mRNA production, $\alpha_{m}$ ). In case (B) the rate of production of an mRNA again increases in proportion to the amount of the preceding protein, but the actual rate is always greater than the baseline rate of mRNA production, $\alpha_{m}$. This second term is similar to that used by Sturrock et al. $(2011,2012)$ in their model of the p53-Mdm2 system. The p53-Mdm2 system may be considered an activator-repressor system, but with the negative feedback being provided by Mdm2enhanced p53 degradation via ubiquitination. In our system, the repression is provided directly by negative feedback to an mRNA. We consider systems which contain a combination of protein repression of mRNA production along with these new terms in which protein activates/promotes mRNA, as such, we refer to them as activator-repressors. A simple schematic of an activator-repressor system with two genes is shown in Figure 9 which can be compared to Figure 4.

\subsection{Two-gene Activator-Repressor: System (A), Simple Activation}

We modify the system of equations for the two-gene repressilator system by altering the Hill function for the 


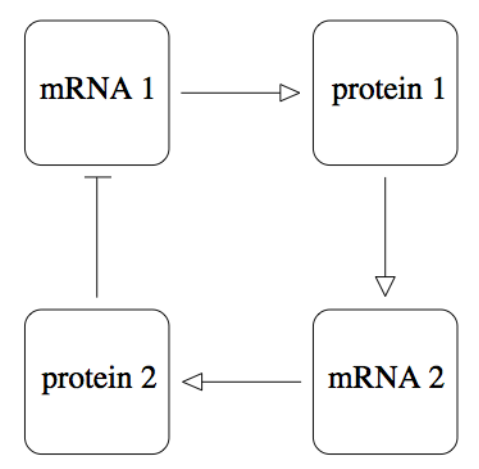

Figure 9: Simple schematic of the two-gene activatorrepressor system. Each species mRNA produces its own protein. Species 1 protein promotes the production of species 2 mRNA, while species 2 protein inhibits the production of species 1 mRNA.

second species so that its mRNA is promoted (rather than inhibited) by the first species protein. We do this first by modifying system (6) to incorporate term the positive feedback term (A), given above, in the equation for the second species' mRNA. As such we refer to this system as activator-repressor system (A). The equations in $1 \mathrm{D}$ are:

$$
\begin{gathered}
\frac{\partial m_{1}}{\partial t}=D_{m} \frac{\partial^{2} m_{1}}{\partial x^{2}}+\frac{\alpha_{m}}{1+p_{2}^{h}} \delta_{x_{m 1}}^{\varepsilon}(x)-\mu_{m} m_{1}, \\
\frac{\partial p_{1}}{\partial t}=D_{p} \frac{\partial^{2} p_{1}}{\partial x^{2}}+\alpha_{p} m_{1} \delta_{x_{p 1}}^{\varepsilon}(x)-\mu_{p} p_{1}, \\
\frac{\partial m_{2}}{\partial t}=D_{m} \frac{\partial^{2} m_{2}}{\partial x^{2}}+\frac{\alpha_{m} p_{1}^{h}}{1+p_{1}^{h}} \delta_{x_{m 2}}^{\varepsilon}(x)-\mu_{m} m_{2}, \\
\frac{\partial p_{2}}{\partial t}=D_{p} \frac{\partial^{2} p_{2}}{\partial x^{2}}+\alpha_{p} m_{2} \delta_{x_{p 2}}^{\varepsilon}(x)-\mu_{p} p_{2},
\end{gathered}
$$

where the $m_{i}(x, t)$ and $p_{i}(x, t)$ are the concentrations of mRNA and protein, respectively for genes $i=\{1,2\}$. The boundary conditions and the initial conditions are, as before (see (7) and (8)).

We solve system (9) with boundary conditions (7) and initial conditions (8) using Matlab and the pdepe solver (comparable results are found with COMSOL). Preliminary investigations of this system show that, as 664 for repressilators, both the separation length between 665 production sites and value of diffusion coefficients 666 are fundamental to the generation of oscillations. 667 Moreover, the ranges for both diffusion coefficients 668 and separation distance remain broadly similar to the 669 repressilator system. In Figure 10 we show results 670 for the two diffusion coefficient regimes and the same 671 four cases as the two-gene repressilator, in order to 672
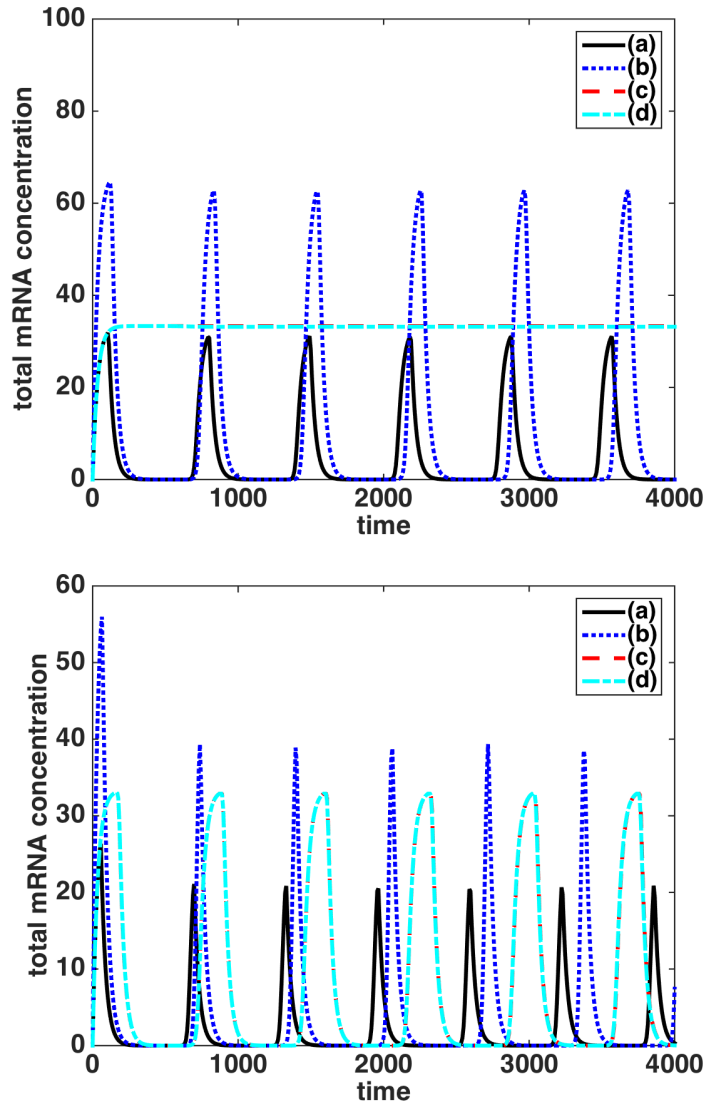

Figure 10: Total mRNA concentrations for species 1 for the two-gene activator-repressor system (A). We consider both the first (top panel) and second (bottom panel) diffusion coefficient regimes. Case (a) - solid black curve: the production sites are $x_{m 1}=x_{m 2}=0.0$ and $x_{p 1}=x_{p 2}= \pm 0.5$. Case (b) - blue dotted curve: the production sites are $x_{m 1}=0.2, x_{m 2}= \pm 0.4, x_{p 1}= \pm 0.7$ and $x_{p 2}= \pm 0.9$. Case (c) - red dashed curve: the production sites are $x_{m 1}=x_{m 2}=0.0, x_{p 1}=x_{p 2}= \pm 0.9$. Case (d) - cyan dot-dashed curve: the production sites are $x_{m 1}=0.0, x_{m 2}= \pm 0.1, x_{p 1}= \pm 0.9$ and $x_{p 2}= \pm 1.0$. Note that in both panels the red curve lies under the cyan curve.

make a direct comparison between the two systems (see Figure 5). For the first diffusion coefficient regime, oscillations occur provided that the separation between the production sites is not too great (in this case oscillating for a separation of 0.5 but not 0.9 ). However, the production sites for the two species are not required to be in the same location; both cases (a) and (b) oscillate. The two-gene activator-repressilator system (A) is a more robust oscillator than the simple 
two-gene repressilator since it will oscillate even when the two genes do not share production sites. For the second diffusion coefficient regime, oscillations occur for all cases. Thus, similarly to the two-gene repressilator system, increasing the diffusion coefficient of mRNA permits oscillations even when there is a greater separation between production sites. In Figure A.18 of Appendix A we show the full space-time behaviour of all species concentrations for the case where $x_{m 1}=x_{m 2}=0.0$ and $x_{p 1}=x_{p 2}= \pm 0.5$. We observe an increase in the overall period of oscillation during which the promoted species persists at high levels for longer while the inhibited gene exhibits oscillations similar to those of Hes1 (and hence the repressilator system.

Since this system oscillates when the species have different production sites we also study what happens when pairs of separation distances between the two species are non-optimum. In Figure 11 we show the behaviour of both mRNAs under the first diffusion coefficient regime for four different cases where separations between mRNAs and proteins are too far apart. In the first two cases the separation on and from one species protein is too far apart. For the first case we consider the protein of species 1 (i.e. $x_{m 1}=x_{m 2}=0.0$, $x_{p 1}= \pm 0.9$ and $\left.x_{p 2}= \pm 0.5\right)$ and for the second the protein of species 2 (i.e. $x_{m 1}=x_{m 2}=0.0, x_{p 1}= \pm 0.5$ and $x_{p 2}= \pm 0.9$ ). Oscillations are observed only in the first case, when the separation on and from species 1 protein is too far apart. In the third and fourth cases the separation on and from one species mRNA is too far apart, for the first case we consider the mRNA of species 1 (i.e. $x_{m 1}=0.0, x_{m 2}= \pm 0.5$ and $\left.x_{p 1}=x_{p 2}= \pm 0.9\right)$ and for the second the mRNA of species 2 (i.e. $x_{m 1}= \pm 0.5$, $x_{m 2}=0.0$ and $x_{p 1}=x_{p 2}= \pm 0.9$ ). Oscillations are observed in both cases.

Interesting dynamics are also observed for the system when we bring the production sites close together. For both diffusion coefficient regimes, we observe large amplitude oscillations in the second species but very low amplitude oscillations in the first species. 725 When only one of the species is too close, it does not 726 matter which species is operating under the optimum 727 separation; oscillations will occur. In either case, the 728 peak widths for species 1 are much narrower and the 729 peak levels obtained are much lower than for species 730 2 , particularly when species 1 is operating under the 731 non-optimum distance. We show this behaviour in 732 Figure 12 which considers three cases of production 733 sites; (a) $x_{m 1}=x_{m 2}=0.0, x_{p 1}=x_{p 2}= \pm 0.1$, (b) 734
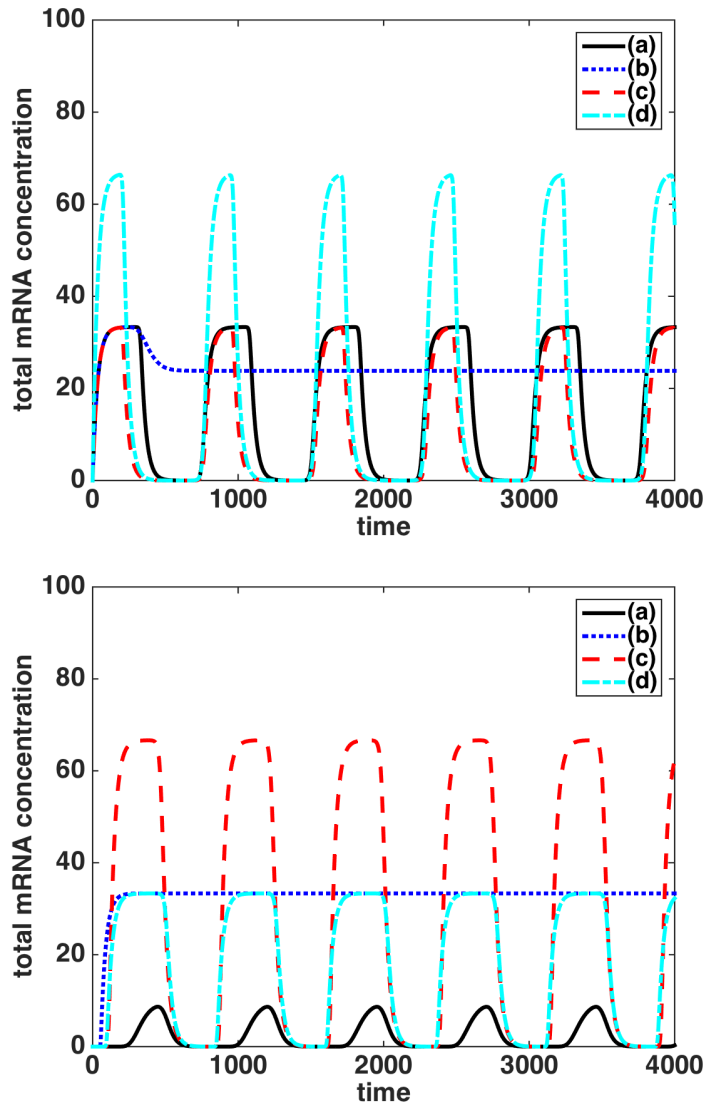

Figure 11: Total mRNA concentrations for species 1 (top panel) and 2 (bottom panel) for the two-gene activator-repressor system (A) under the first diffusion coefficient regime, $D_{m}=D_{p}=D$. Case (a) - solid black curve: the production sites are $x_{m 1}=x_{m 2}=0.0$, $x_{p 1}= \pm 0.9$ and $x_{p 2}= \pm 0.5$. Case (b) - dotted blue curve: the production sites are $x_{m 1}=x_{m 2}=0.0$, $x_{p 1}= \pm 0.5$ and $x_{p 2}= \pm 0.9$. Case (c) - dashed red curve: the production sites are $x_{m 1}=0.0, x_{m 2}= \pm 0.5$ and $x_{p 1}=x_{p 2}= \pm 0.9$. Case $(\mathrm{d})$ - dot-dashed cyan curve: the production sites are $x_{m 1}= \pm 0.5, x_{m 2}=0.0$ and $x_{p 1}=x_{p 2}= \pm 0.9$.

$x_{m 1}=x_{m 2}=0.0, x_{p 1}= \pm 0.1$ and $x_{p 2}= \pm 0.5$ and (c) $x_{m 1}=x_{m 2}=0.0, x_{p 1}= \pm 0.5$ and $x_{p 2}= \pm 0.1$. Oscillations are observed in all cases, although the amplitude of species 1 oscillations for case (a) is extremely low.

The findings presented in this section indicate that the two-gene activator-repressor system (A) is a more robust oscillator than its repressilator counterpart and will oscillate for a wide range of conditions like the three-gene repressilator. However, it remains important 

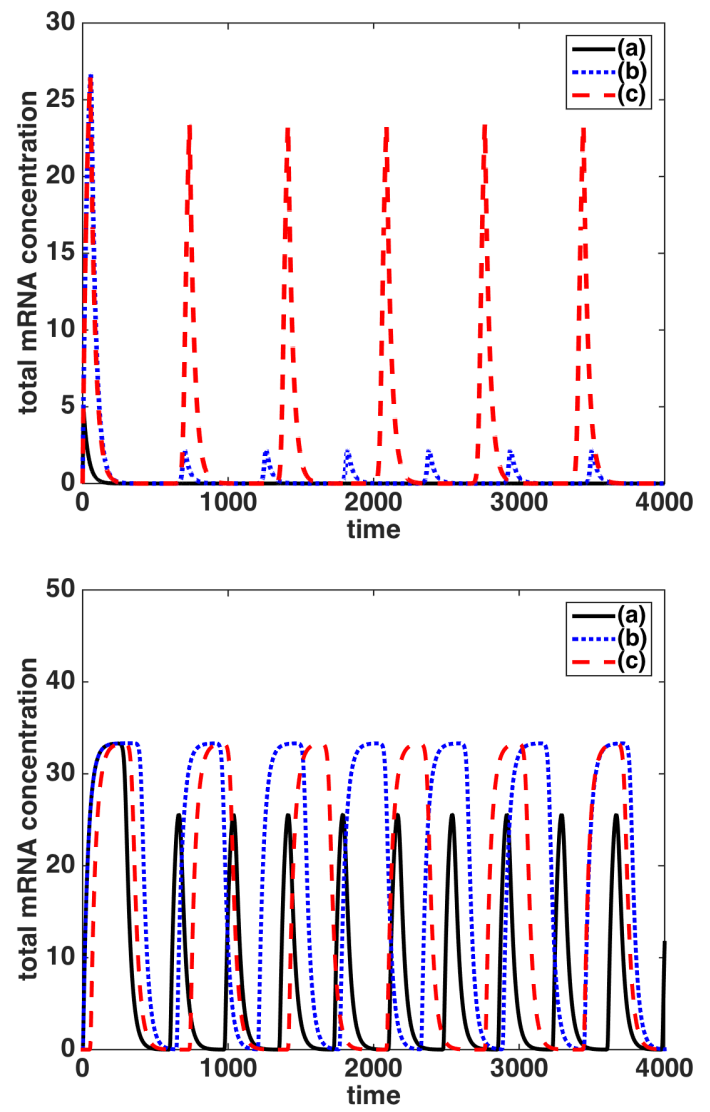

Figure 12: Total mRNA concentrations for species 762 1 (top panel) and 2 (bottom panel) for the two-gene activator-repressor system (A) under the first diffusion ${ }_{765}$ coefficient regime, $D_{m}=D_{p}=D$. Case (a) - solid ${ }_{766}$ black curve: the production sites are $x_{m 1}=x_{m 2}=0.0,{ }_{767}$ $x_{p 1}=x_{p 2}= \pm 0.1$. Case (b) - dotted blue curve: the 768 production sites are $x_{m 1}=x_{m 2}=0.0, x_{p 1}= \pm 0.1$ and 769 $x_{p 2}= \pm 0.5$. Case (c) - dashed red curve: the production 770 sites are $x_{m 1}=x_{m 2}=0.0, x_{p 1}= \pm 0.5$ and $x_{p 2}= \pm 0.1$.

$$
\begin{array}{r}
\frac{\partial m_{1}}{\partial t}=D_{m_{1}} \frac{\partial^{2} m_{1}}{\partial x^{2}}+\frac{\alpha_{m}}{1+p_{2}^{h}} \delta_{x_{m_{1}}}^{\varepsilon}(x)-\mu_{m} m_{1}, \\
\frac{\partial p_{1}}{\partial t}=D_{p_{1}} \frac{\partial^{2} p_{1}}{\partial x^{2}}+\alpha_{p} m_{1} \delta_{x_{p_{1}}}^{\varepsilon}(x)-\mu_{p} p_{1}, \\
\frac{\partial m_{2}}{\partial t}=D_{m_{2}} \frac{\partial^{2} m_{2}}{\partial x^{2}}+\left[\alpha_{m}+\frac{\beta_{m} p_{1}^{h}}{1+p_{1}^{h}}\right] \delta_{x_{m_{2}}}^{\varepsilon}(x)-\mu_{m} m_{2}, \\
\frac{\partial p_{2}}{\partial t}=D_{p_{2}} \frac{\partial^{2} p_{2}}{\partial x^{2}}+\alpha_{p} m_{2} \delta_{x_{p_{2}}}^{\varepsilon}(x)-\mu_{p} p_{2},
\end{array}
$$

where the variables $m_{i}(x, t)$ and $p_{i}(x, t)$ remain as per (9). The boundary conditions and the initial conditions are, as before (see (7) and (8)).

Once again we explore the effect of varying the distance between the mRNA gene-site and the protein production sites on the spatio-temporal dynamics of the system. We take $\beta_{m}=10.0$ while all other parameters are as in Table 1. Figure 13 shows the computational results of numerical simulations of (10) where the locations of the protein production sites are varied relative to the fixed mRNA gene-sites $\left(x_{m i}=0\right)$. As before we solve the system (10) with associated boundary conditions (7) and initial conditions (8) using Matlab and the pdepe solver (comparable results were found using COMSOL). The graphs show the total concentrations of mRNA and protein for species 1 (comparable behaviour for species 2) over time and demonstrate that if the protein production sites are too close or too far away from the mRNA gene-site, then oscillations are lost. We note that although there is a range of values of $x_{p i}$ which lead to oscillations, this range is far more constricted and further away from the mRNA gene-site than for the previous synthetic systems. In this case a separation of 0.5 length units would be too close, while a separation of 0.9 length units would be optimal. Furthermore, this system is far more sensitive to changes in diffusion coefficients. While for the other two systems we were able to vary the diffusion coefficients by orders of magnitude, for this system only slight changes may be implemented. As such we do not consider the second diffusion coefficient regime for this system. When oscillations do occur we observe that the nature of the oscillations is also different; while peak levels of mRNA and protein are comparable to levels seen for the other systems, the baseline values of both species are far higher. This is intuitive since for this activator-repressor system (B), the mRNA production rate (of one species) now varies between a lower bound of $\alpha_{m}$ and an upper bound of $\alpha_{m}+\beta_{m}$, whereas for activator-repressor system (A), both mRNA production rates are bounded below by zero 

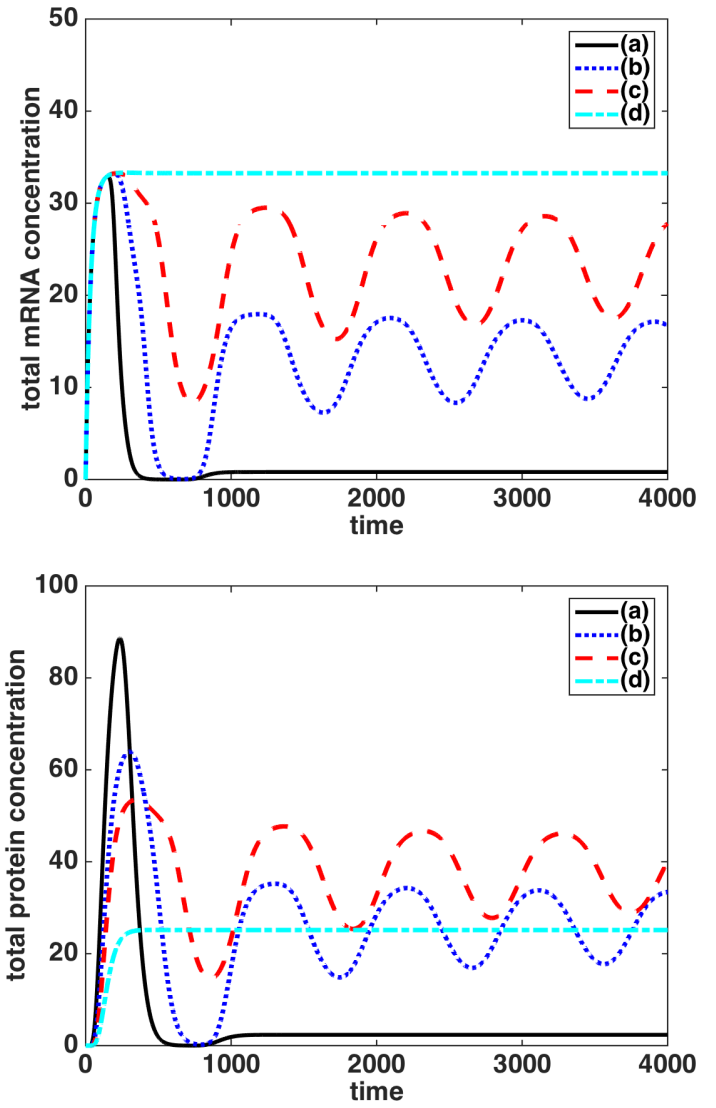

Figure 13: Total mRNA (top panel) and protein (bottom panel) concentration for species 1 for the two-gene ${ }_{827}$ activator-repressor system (B). The mRNA gene-sites are $x_{m_{i}}=0.0, i=1,2$ and the protein production sites ${ }_{829}$ where are $x_{p_{i}}= \pm 0.8, \pm 0.88, \pm 0.94, \pm 1.0, i=1,2$. Results are for the first diffusion coefficient regime, $D_{m}={ }_{831}$ $D_{p}=D$.

and above by $\alpha_{m}$. This behaviour can also be seen in Figure A.19 of Appendix A which shows the full spacetime behaviour of all species concentrations for the case where $x_{m 1}=x_{m 2}=0.0$ and $x_{p 1}=x_{p 2}= \pm 0.9$. We observe sustained oscillations of mRNA and protein for both species in space and time, with a sustained base level of expression.

\subsection{Three-gene Activator-Repressor Systems}

To consider the activator-repressor systems further, we 845 make preliminary investigations into three-gene sys- 846 tems. Note that the addition of a further gene to ${ }_{847}$ activator-repressor systems increases the complexity. 848 An activator-repressor system with three-genes could 849

$$
31 \text { }
$$

contain either two up-regulated and one down-regulated mRNA or two down-regulated and one up-regulated mRNA. Our brief investigations show that (for either activator-repressor system) a system with two downregulated mRNA will not oscillate for a range of conditions but a system with only one-down regulated mRNA will always lead to oscillations in at least one of the species concentrations.

\section{Discussion and Conclusions}

In this paper we have considered spatio-temporal models of gene regulatory networks considering both actual (Hes1) and synthetic (repressilators and acitvator-repressors) systems. The study of synthetic GRNs is relevant in the current climate of research as biologists collaborate with mathematicians to construct and analyse such systems to gain a deeper understanding of the underpinning biology (see, for example, Balagadde et al., 2008; Becskei and Serrano, 2000; Elowitz and Leibler, 2000; Purcell et al., 2010; Chen et al., 2012; O'Brien et al., 2012; Yordanov et al., 2014). It is known that GRNs with negative feedback components frequently exhibit oscillatory behaviour and mathematical modelling has largely focussed on the temporal dynamics using ODE and/or DDE models. Our results show that the dynamics of GRNs can be controlled by spatial components of the PDE model with specific spatial conditions leading to oscillations. This work is in-line with and generalises previous work by Sturrock et al. $(2011,2012)$ and Chaplain et al. (2015). We stress the importance of including spatial components when modelling GRNs, as they are key to generating periodic behaviour.

More specifically we have investigated the importance of gene and protein location by considering the relative positions of mRNA gene-sites and protein production sites. We have found that the separation between mRNA and protein production for the simple Hes1 system must be optimised in order to achieve oscillations. This optimisation requires the protein production sites to be neither too far away nor too close to the mRNA gene-site, although the precise optimal ranges will be affected by the size of the diffusion coefficient(s). Imayoshi and Kageyama (2014) have shown that oscillatory and sustained expression of bHlH transcription factors (such as Hes1) correspond to different states for neural progenitors (self-renewing and fate determining, respectively). Changes to spatial structure provide a realistic mechanism for control of GRNs. Since parameters, diffusion coefficients in 
particular, are not well known, we suggest further 902 work is needed to determine parameter regimes more 903 precisely.

Our investigation into synthetic networks confirms 906 the importance of spatial modelling of GRNs whilst at 907 the same time has delivered some interesting results 908 which merit further investigation and analysis.

Firstly we have found that multi-gene systems 911 built solely on negative feedback (repressilators) 912 preferentially generate oscillations for systems with 913 an odd number of genes. In particular systems with 914 three or five genes are found to oscillate for a wide 915 range of conditions whereas systems with two or four 916 do not. This disparity in behaviour has been found in ${ }_{917}$ other models (see Purcell et al., 2010). For three- (and 918 five-gene) systems, our simulations showed that dispar- 919 ate production sites for each species caused elongated 920 oscillation periods (particularly the time spent at peak ${ }_{921}$ concentration levels) compared to cases where all spe- 922 cies shared production sites. Oscillations were found 923 provided that the greatest separation between mRNA 924 gene-site and protein production site was optimised. 925 Initial investigations of higher order systems indicates 926 that increasing the number of genes makes the system 927 more robust and likely to oscillate. For an ODE model 928 of multi-gene repressilators, Strelkowa and Barahona 929 (2010) found that increasing the number of genes lead 930 to increased stability of periodic solutions. Our results 931 indicate that this is also true of a comparable PDE ${ }_{932}$ system, something which requires further study. If 933 proven, this result may suggest that the complexity 934 due to the number of genes in biologically realistic 935 networks, particularly cascades, are designed in such a 936 way to maximise the likelihood of periodic behaviour ${ }_{937}$ while the precise locations of processes may be the key 938 to controlling the timescale of oscillations.

Our study of activator-repressor models considered two such systems: the first, (A), in which the promotion of one species is activated by another but remains 943 capped by the natural rate of mRNA production (which 944 serves as an upper bound) and the second, (B), where 945 production is enhanced above the natural rate of mRNA 946 production (now serving as a lower bound). The 947 first of these two systems is more likely to oscillate 948 than the second which is in agreement with previous 949 modelling (Sturrock et al., 2015). In fact the two-gene 950 activator-repressor (A) oscillates more readily than the 951 two-gene repressilator (particularly when production 952 sites of the species are different). Again oscillations 953 were found provided that the greatest separation between mRNA gene-site and protein production site was optimised. Very brief investigations into three-gene activator-repressor systems have shown that oscillations are unlikely when two species inhibits and one promotes for either system (A) or (B). Oscillations are found when one species inhibits and two promote (particularly for activator-repressor system (A)). This result and others discussed here provide some indication that oscillatory behaviour is governed to an extent by the global "sign" of the feedback. For example, a two-gene repressilator is globally positive (having two negative interactions), while a two-gene activator-repressor is globally negative (having one negative and one positive interaction). For the results presented here we have found that globally negative systems oscillate preferentially. However, this is merely an observation and requires further study. Increasing the number of genes/species for systems with both activation and repression leads to increased variation in the system set-up (depending on how many and which species activate or inhibit). As such they have not been well-studied. We have provided some key simulations, however, further work on such systems is required and is on-going.

In this paper we have focussed only on the behaviour of intracellular gene regulation in isolation within a single cell. Of course in many in vitro experiments and in vivo, cells exist in communities and it is important that they communicate with and signal to each other. For example, as is the case for the developmental process of somitogenesis, intracellular signalling is coordinated in an intercellular manner. In particular the oscillatory expression of certain proteins within cells can be synchronous (see, for example, Lewis, 2003; Terry et al., 2011). Understanding how cell-cell interactions affect gene regulation and the dynamics of a group of cells or a tissue could form the basis of future investigation.

Overall the results of this paper confirm the importance of modelling transcription factor systems where negative feedback loops are involved (both actual and synthetic), using explicitly spatial models. Given the current level of interest in synthetic biology and the technological tools available to synthetic biologists, the findings in this paper indicate that experimentalists should take molecular movement into account when trying to design such systems.

Most of the previous work in this area has adop- 
ted a delay differential equation approach where a 1006 discrete time delay is included in a system of ODEs. 1007 As well as transcription and translation, the delay is 1008 taken to account for molecular movement without 1009 explicitly incorporating this mechanism into the model. 1010 By explicitly incorporating spatial terms into our 1011 model, we are able to say something more focussed 1012 about the importance of molecular movement and the 1013 effect of molecular transport time between nucleus 1014 and cytoplasm. Recent work on spatial models and 1015 the results of this paper show that the incorporation 1016 of spatial affects into models of GRNs allows us to 1017 reproduce the known oscillatory dynamics, periodicity 1018 being an emergent property of the PDE systems. While 1019 delay equations also capture the overall oscillatory dynamics of GRNs, considering spatial models which incorporate intracellular molecular movement directly will allow connections to be made with experimental 1021 data arising from single cell experiments. Increasingly 1022 biologists are developing techniques to tag and monitor ${ }_{1023}$ the movement of molecules in single cells (e.g. FRAP, ${ }_{1024}$ FLIP, FRET, FLIM, FCS, FCCS, ICS, ICCS, PCA 1025 etc. cf. Spiller et al. (2010)). Developing appropriate ${ }_{1026}$ mathematical models that have the ability to analyse ${ }_{1027}$ the spatial data that arises from such single-cell ex- 1028 periments is also, therefore, important (Spiller et al., ${ }_{1029}$ 2010), which is where spatial models such as those 1030 presented in this paper can bring new insights to 1031 the problem. In addition to describing the overall ${ }_{1032}$ mRNA and protein concentrations over time (cf. Lewis 1033 (2003)) the computational results of our model (i.e. 1034 the spatio-temporal figures in Appendix A) may be 1035 compared with single cell experiments where proteins 1036 are fluorescently labelled, (e.g. Lahav et al., 2004; 1037 Nelson et al., 2004; Geva-Zatorsky et al., 2006; Ashall 1038 et al., 2009; Spiller et al., 2010), although this is beyond ${ }_{1039}$ the remit of the current paper. Populations of cells are 1040 heterogenous in nature, with differences at both the 1041 genetic and the phenotypic level. In order to continue 1042 to study intracellular dynamics (and potentially the ${ }_{1043}$ subsequent cell-cell dynamics) it is important to have 1044 mathematical models which can account explicitly 1045 for the phenotypic variation between individual cells. 1046 Spatial models permit a realistic modelling of indi- 1047 vidual cells where it is required to analyse the aspects 1048 of phenotypic variation which arise from differences 1049 in intracellular structure - such as different protein 1050 production sites, variations in diffusion coefficients 1051 between molecules, spatially-dependent diffusion due ${ }_{1052}$ to intracellular structural heterogeneity, or transport 1053 of molecules across the nuclear membrane. As the 1054 imaging techniques themselves are further developed 1055 and refined (e.g. Betzig et al., 2006; Manley et al., 2008; van de Linde et al., 2011; Won et al., 2011; Bar-On et al., 2012; Hiersemenzel et al., 2013) it is also important to continue to develop spatial mathematical models.

A follow-up paper will discuss similar results for a stochastic model. Taking the lead from Sturrock et al. (2013) we will examine repressilator and activatorrepressor systems by modelling their biochemical reactions, incorporating the idea of a promoter to which the protein species bind/unbind affecting the rate of mRNA transcription. Spatial aspects will also be incorporated to reaffirm the message relayed here.

\section{Appendix A. Spatio-temporal Figures}

Here we provide the full spatio-temporal behaviour of mRNA and protein concentrations for certain cases highlighted throughout the paper, which while not adding to the results compound them and help to show some of the key types of behaviour observed.

In Figure A.14 we show the full space-time behaviour of mRNA and protein concentrations for the Hes 1 system (1) with $x_{m}=0.0$ and $x_{p}=\{ \pm 0.1, \pm 0.5, \pm 0.9\}$, in the top, middle and bottom panels, respectively.

In Figure A.15 we show the full space-time behaviour of mRNA and protein concentrations for the Hes1 system (1) with $x_{m}=0.0$ and $x_{p}=\{ \pm 0.1, \pm 0.5, \pm 0.9\}$, for the second diffusion coefficient regime. Thus, we enable direct comparisons between Figure A.15 (in which $D_{m}=0.0075$ and $D_{p}=D$ ) and Figure A.14 (in which $D_{m}=D_{p}=D$ ). We note that for the second diffusion coefficient regime peak levels of mRNA are typically lower, while peak levels of the protein are typically higher.

In Figure A.16 we show the full space-time behaviour of mRNA and protein concentrations for the three-gene $(n=3)$ repressilator system (6) with $x_{m i}=0.0$ and $x_{p i}= \pm 0.5$. We observe that all three species exhibit the same behaviour (the space-time plots in Figure A.16 are identical for each species). Furthermore, this behaviour matches exactly with the behaviour of the Hes1 system with $x_{p}= \pm 0.5$ (the space-time plots in Figure A.16 are identical to those in the middle panel of Figure A.14).

In Figure A.17 we show the full space-time behaviour of mRNA and protein concentrations for the three-gene $(n=3)$ repressilator system (6) with $x_{m 1}=0.0$, 

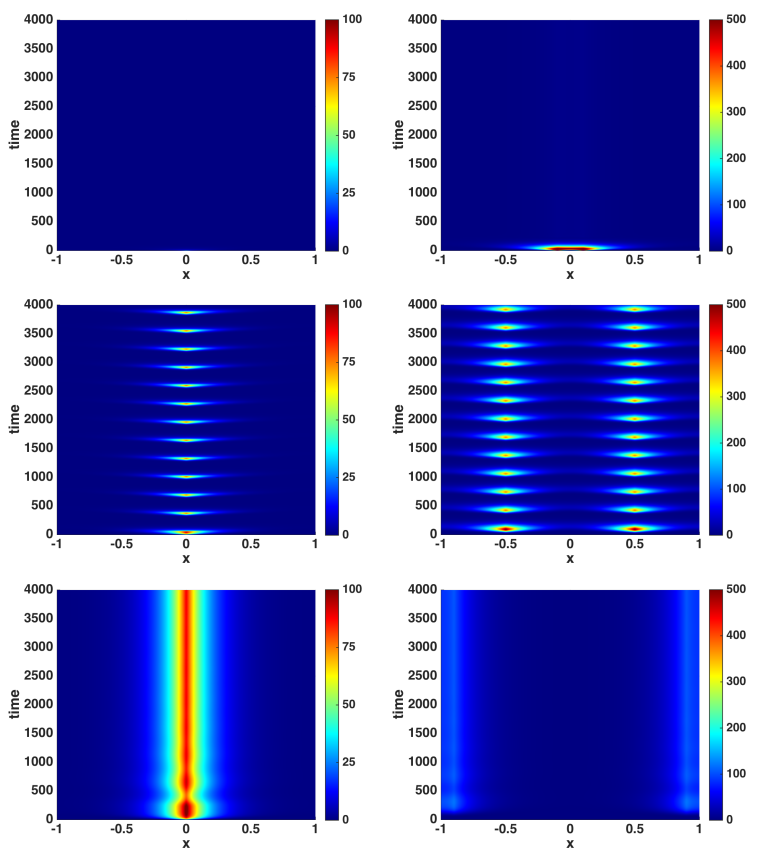

Figure A.14: Spatial-temporal concentrations of mRNA (left) and protein (right) concentrations for the Hes 1 system. The top panels indicate the behaviour when the production sites are too close together $\left(x_{m}=0.0\right.$ and $x_{p}= \pm 0.1$ ). The middle panels indicate the behaviour when the production sites are at an optimum distance $\left(x_{m}=0.0\right.$ and $\left.x_{p}= \pm 0.5\right)$. The bottom panels indicate the behaviour when the production sites are too far apart $\left(x_{m}=0.0\right.$ and $\left.x_{p}= \pm 0.9\right)$.
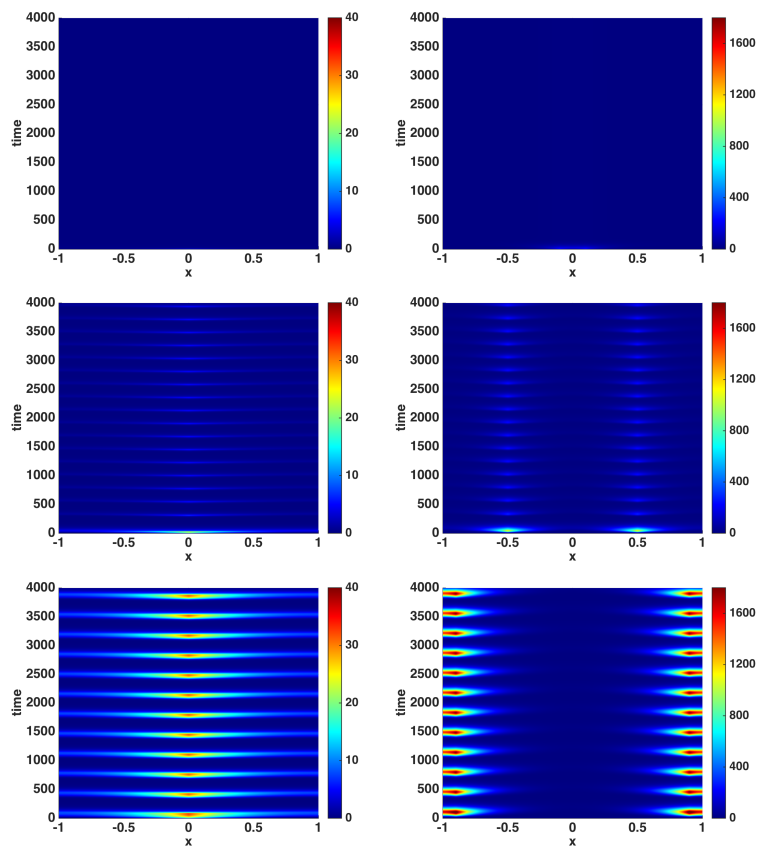

Figure A.15: Spatial-temporal concentrations of mRNA (left) and protein (right) concentrations for the Hes1 system, with $D_{m}=0.0075$ and $D_{p}=D$. The top panel indicate the behaviour when the production sites are $x_{m}=0.0$ and $x_{p}= \pm 0.1$. The middle panels indicate the behaviour when the production sites are $x_{m}=0.0$ and $x_{p}= \pm 0.5$. The bottom panels indicate the behaviour when the production sites are $x_{m}=0.0$ and $x_{p}= \pm 0.9$.

In Figure A.19 we show the full space-time behaviour of all species concentrations for the case where $x_{m 1}=x_{m 2}=0.0$ and $x_{p 1}=x_{p 2}= \pm 0.9$. We observe sustained oscillations of mRNA and protein for both species in space and time, with a sustained base level of expression. Note that peak levels are higher for species 2.

\section{Appendix B. Hes1 System in 2D and 3D}

We also considered the behaviour of the Hes 1 system in other geometries. In considering production zones for the mRNA and protein, we discovered behaviour comparable to the $1 \mathrm{D}$ case. The production zones for mRNA and protein must be optimally separated if the system is to oscillate. The results for these other geometries show that the results reported in the paper are independent of the geometry. 

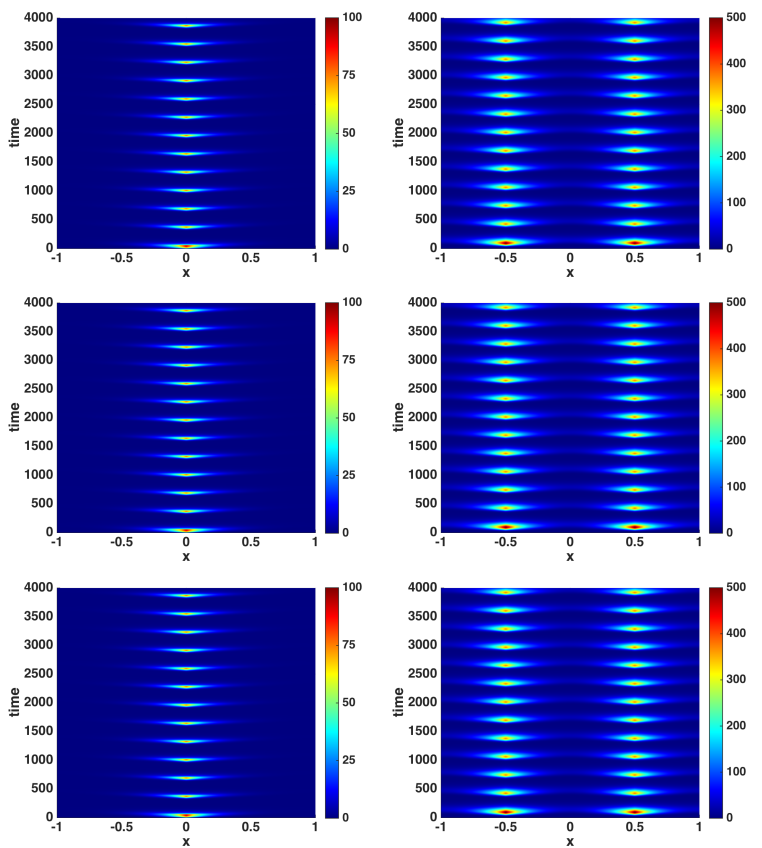

Figure A.16: Spatial-temporal concentrations of mRNA (left) and protein (right) for the three-gene repressilator with species $1-3$ displayed in the top to bottom panels, respectively. Shown is the behaviour when the production sites are in the same location, namely $x_{m 1}=x_{m 2}=$ $x_{m 3}=0.0$ and $x_{p 1}=x_{p 2}=x_{p 3}= \pm 0.5$. Results are for the first diffusion coefficient regime, $D_{m}=D_{p}=D$.

\section{Appendix B.1. Hes1 System for a Unit Circle}

We model the cell as a 2D circular domain with radius unity, centered at the origin. The system was given by the following equations:

$$
\begin{gathered}
\frac{\partial m}{\partial t}=D \nabla^{2} m+\frac{\alpha_{m}}{1+p^{h}} \delta_{r_{m}}^{\varepsilon}(x, y)-\mu m \\
\frac{\partial p}{\partial t}=D \nabla^{2} p+\alpha_{p} m \delta_{r_{p}}^{\varepsilon}(x, y)-\mu p
\end{gathered}
$$

where $m(x, y, t)$ is the concentration of hes 1 mRNA and $p(x, y, t)$ is the concentration of Hes 1 protein. The 1112 boundary conditions at the cell membrane are:

$$
\frac{\partial m}{\partial \mathbf{n}}=\frac{\partial p}{\partial \mathbf{n}}=\mathbf{0}
$$

where $\mathbf{n}$ is a unit normal to the boundary surface. We ${ }_{1117}$ assume zero initial concentrations. We consider that the 1118 mRNA and protein are made in specific regions of the 1119 cell, and specifically consider the separation between 1120 these two regions. We use a comparable Dirac approx- 1121 imations of the $\delta$-like distribution function located at the 1122
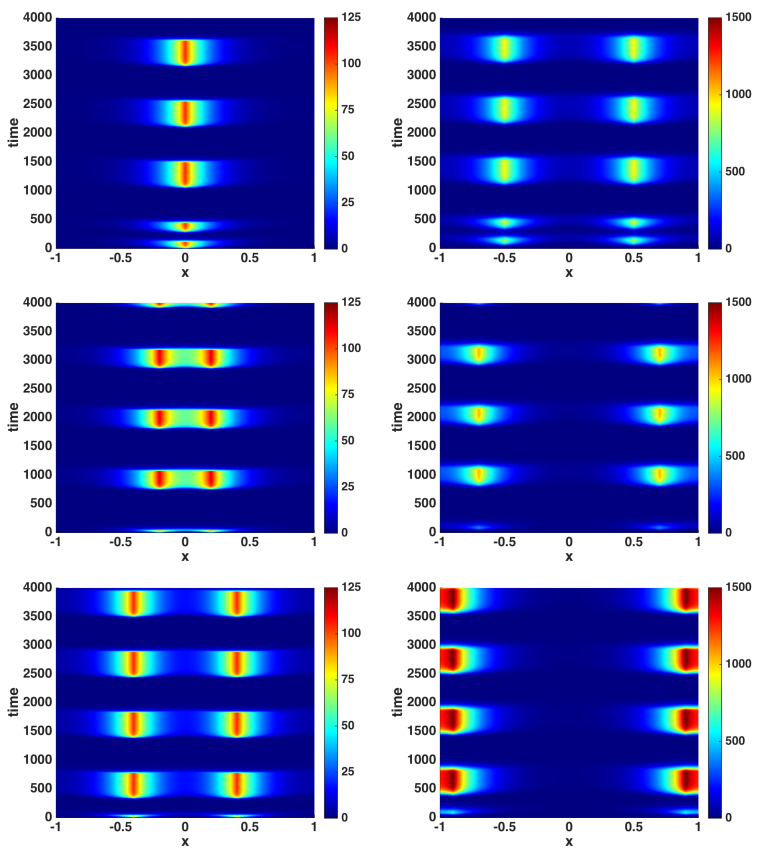

Figure A.17: Spatial-temporal concentrations of mRNA (left) and protein (right) for the three-gene repressilator with species $1-3$ displayed in the top to bottom panels, respectively. Shown is the behaviour when the production sites are in different locations, namely $x_{m 1}=0.0$, $x_{m 2}= \pm 0.2, x_{m 3}= \pm 0.4, x_{p 1}= \pm 0.5, x_{p 2}= \pm 0.7$ and $x_{p 3}= \pm 0.9$. Results are for the first diffusion coefficient regime, $D_{m}=D_{p}=D$.

production sites radii $r_{i}$, where $i=\{m, p\}$, such that

$$
\delta_{r_{i}}^{\varepsilon}(x, y)= \begin{cases}\frac{1}{2 \varepsilon}\left[1+\cos \left(\frac{\pi\left(r-r_{i}\right)}{\varepsilon}\right)\right] & \left|r-r_{i}\right|<\varepsilon, \\ 0 & \left|r-r_{i}\right| \geq \varepsilon,\end{cases}
$$

with $r$ the radial position, such that $r^{2}=x^{2}+y^{2}$. As such, $r_{m} \pm \varepsilon$ and $r_{p} \pm \varepsilon$ will be annular regions of mRNA and protein production respectively. We considered the effect of varying the position of $r_{m}$ and $r_{p}$ finding that there must be an optimum separation between $r_{m}$ and $r_{p}$. There must be a gap between sites where mRNA and protein are produced but this gap must not be too great.

In Figure B.20 we show the behaviour of the mRNA and protein concentrations for specific cases. In each case $r_{m}=0.0$ is fixed and we vary $r_{p}$ taking the values $r_{p}=\{0.1,0.3,0.5,0.7,0.9\}$. We solve system (B.1) using COMSOL taking the same parameters as for the 1D model (see Table 1) apart from $\alpha_{m}$ which we increase to 10.0 to account for the increased domain size going 

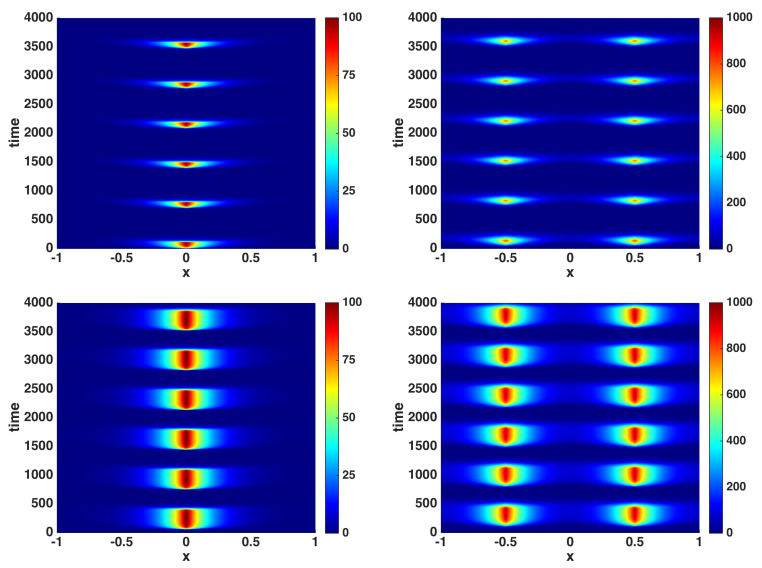

Figure A.18: Spatial-temporal concentrations of mRNA (left) and protein (right) for the two-gene activatorrepressor system (A) with species 1 (top panel) and 2 (bottom panel). Shown is the behaviour when the production sites are in the same location and optimally separated, namely, $x_{m 1}=x_{m 2}=0.0$ and $x_{p 1}=x_{p 2}= \pm 0.5$. Results are for the first diffusion coefficient regime, $D_{m}=D_{p}=D$. from $1 \mathrm{D}$ to $2 \mathrm{D}$. When the gap between production is too small $\left(r_{p}=0.1\right)$ or too great $\left(r_{p}=0.9\right)$ the system does not oscillate. For values of $r_{p}$ in between (i.e. for optimally separated production zones) the system shows periodic behaviour. Qualitatively this behaviour is as for the 1D system as can be seen be comparing Figures B.20 and 2.

\section{Appendix B.2. Hes1 System for an Ellipse}

We model the cell in 2D as a elliptical domain, centered ${ }^{1153}$ at the origin. The semi-major axis is taken to be $1.5{ }^{1154}$ while the semi-minor axis is set to unity. The delta-like ${ }^{1155}$ functions in this geometry take the same form but where ${ }^{1156}$ now

$$
r^{2}=\frac{x^{2}}{a^{2}}+\frac{y^{2}}{b^{2}}
$$

with $a=1.5$ and $b=1$ to mirror the shape of the ${ }_{1161}$ elliptical domain. Again whether the system will 1162 oscillate depends on the values of $r_{m}$ and $r_{p}$, more ${ }_{1163}$ specifically on their relative position.

In Figure B. 21 we show the behaviour of the mRNA and protein for specific cases to compare with Fig- ${ }_{1167}$ ure B.20. In each case $r_{m}=0.0$ is fixed and we vary 1168 $r_{p}$ taking the values $r_{p}=\{0.1,0.3,0.5,0.7,0.9\}$. Again 1169 we solve system (B.1) using COMSOL and take the 1170 same parameters as for the 2D circular model. The 1171
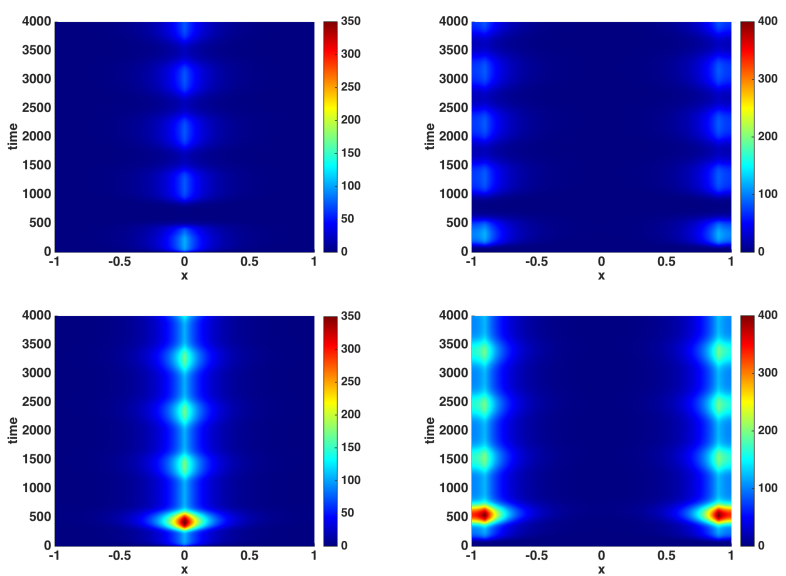

Figure A.19: Spatial-temporal concentrations of mRNA (left) and protein (right) for the two-gene activatorrepressor system (B) with species 1 (top panel) and 2 (bottom panel). Shown is the behaviour when the production sites are in the same location and optimally separated, namely, $x_{m 1}=x_{m 2}=0.0$ and $x_{p 1}=x_{p 2}= \pm 0.9$. Results are for the first diffusion coefficient regime, $D_{m}=D_{p}=D$.

results are similar to the $1 \mathrm{D}$ and circular $2 \mathrm{D}$ cases, although oscillations are not sustained for the elliptical case when $r_{p}=0.7$. However, the result that there must be an optimum separation between production zones is still valid.

\section{Appendix B.3. Hes1 System for a Sphere}

We model the cell in 3D as a spherical domain, centered at the origin with radius unity. The equations we use in this geometry are equivalent to (B.1) but now we consider three dimensions so that $m(x, y, z, t)$ is the concentration of hes 1 mRNA and $p(x, y, z, t)$ is the concentration of Hes1 protein. The delta-like functions in this geometry take the same form but where now $r^{2}=x^{2}+y^{2}+z^{2}$. As before whether the system will oscillate depends on the separation between $r_{m}$ and $r_{p}$.

In Figure B.22 we show the behaviour of the mRNA and protein for specific cases to compare with Figures B.20 and B.21. In each case $r_{m}=0.0$ is fixed and we vary $r_{p}$ taking the values $r_{p}=\{0.1,0.3,0.5,0.7,0.9\}$. Again we solve the system (B.1) using COMSOL and take the same parameters as for the 2D circular model, although to avoid difficulties with the mesh size we choose $\varepsilon=0.1$. The results are comparable to the 1D and $2 \mathrm{D}$ cases. 

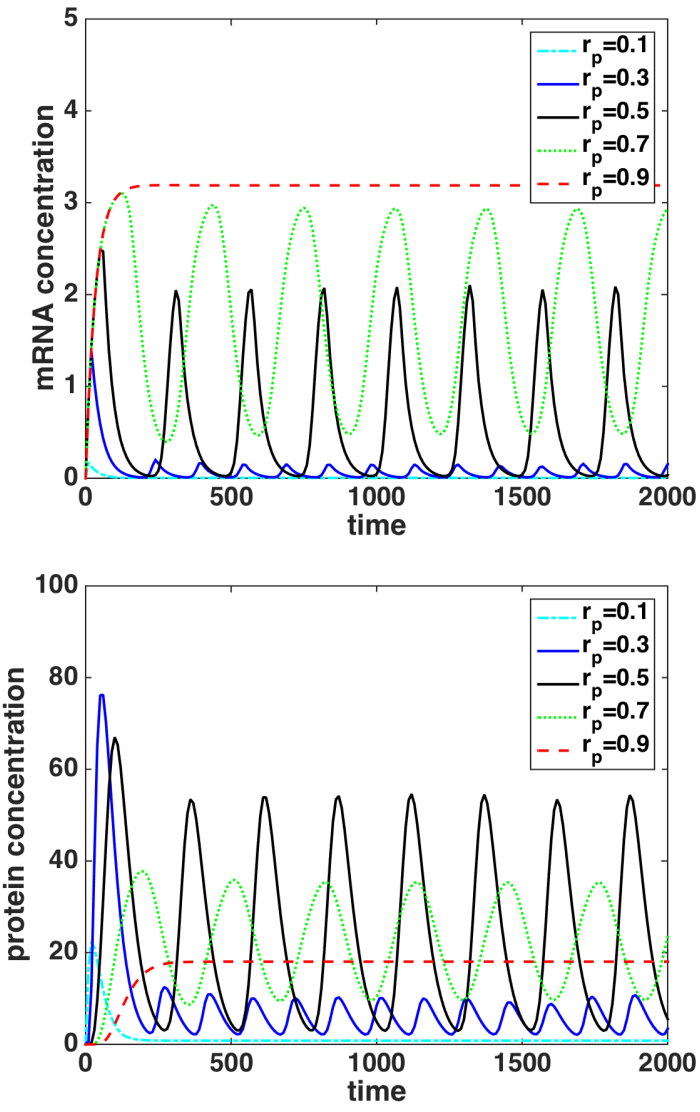

Figure B.20: Total mRNA (top panel) and protein (bottom panel) concentrations for the Hes1 system (B.1) in 2D with circular geometry. Shown is the behaviour when the mRNA gene-site is located at $r_{m}=0.0$ and the protein production zones, $r_{p}$, vary (see legend).

\section{References}

Ashall, L., Horton, C. A., Nelson, D. E., Paszek, P., Harper, C. V., 1194 Sillitoe, K., Ryan, S., Spiller, D. G., Unitt, J. F., Broomhead, D. S., 1195 Kell, D. B., Rand, D. A., Sée, V., White, M. R. H., 2009. Pulsatile 1196 stimulation determines timing and specificity of nf-kb-dependent 1197 transcription. Science 324, 242-246. Balagadde, F. K., Song, H., Ozaki, J., Collins, C. H., Barnet, M., 1199 Arnold, F. H., Quake, S. R., You, L., 2008. A synthetic escherichia 1200 coli predator-prey ecosystem. Mol. Syst. Biol. 4:187.

Bar-On, D., Wolter, S., van de Linde, S., Heilemann, M., Nudelman, 1202 G., Nachliel, E., Gutman, M., Sauer, M., Ashery, U., 2012. Super- 1203 resolution imaging reveals the internal architecture of nano-sized 1204 syntaxin clusters. J Bio Chem 287, 27158-27167. Becskei, A., Serrano, L., 2000. Engineering stability in gene networks 1206 by autoregulation. Nature 405, 590-593. Bernard, S., Čajavec, B., Pujo-Menjouet, L., Mackey, M. C., Herzel, 1208 H., 2006. Modeling transcriptional feedback loops: The role of 1209 gro/tle1 in hes 1 oscillations. Philos. Trans. A. Math. Phys. Eng. 1210 Sci. 15, 1155-1170.

1198

1201

1205

nych, S., Bonifacino, J. S., Davidson, M. W., Lippincott-Schwartz, J., Hess, H. F., 2006. Imaging intracellular fluorescent proteins at nanometer resolution. Science 313, 1642-1645.

Busenberg, S., Mahaffy, J. M., 1985. Interaction of spatial diffusion and delays in models of genetic control by repression. J. Math. Biol. 22, 313-333.

Cangiani, A., Natalini, R., 2010. A spatial model of cellular molecular trafficking including active transport along microtubules. J. Theor. Biol. 267, 614-625.

Chaplain, M. A. J., Ptashnyk, M., Sturrock, M., 2015. Hopf bifurcation in a gene regulatory network model: Molecular movement causes oscillations. Math. Mod. Meth. Appl. S. 25 (6), 1179-1215.

Chen, Y. Y., Galloway, K. E., Smolke, C. D., 2012. Synthetic biology: advancing biological frontiers by building synthetic systems. Genome Biol. 13:240.

Dimitrio, L., Clairambault, J., Natalini, R., 2013. A spatial physiological model for p53 intracellular dynamics. J. Theor. Biol. 316, 69-24.

Eliaš, J., Clairambault, J., 2014. Reaction-diffusion systems for spatio-temporal intracellular protein networks: a beginner's guide with two examples. Comp. Struct. Biotechnol. J. 10, 14-22. 

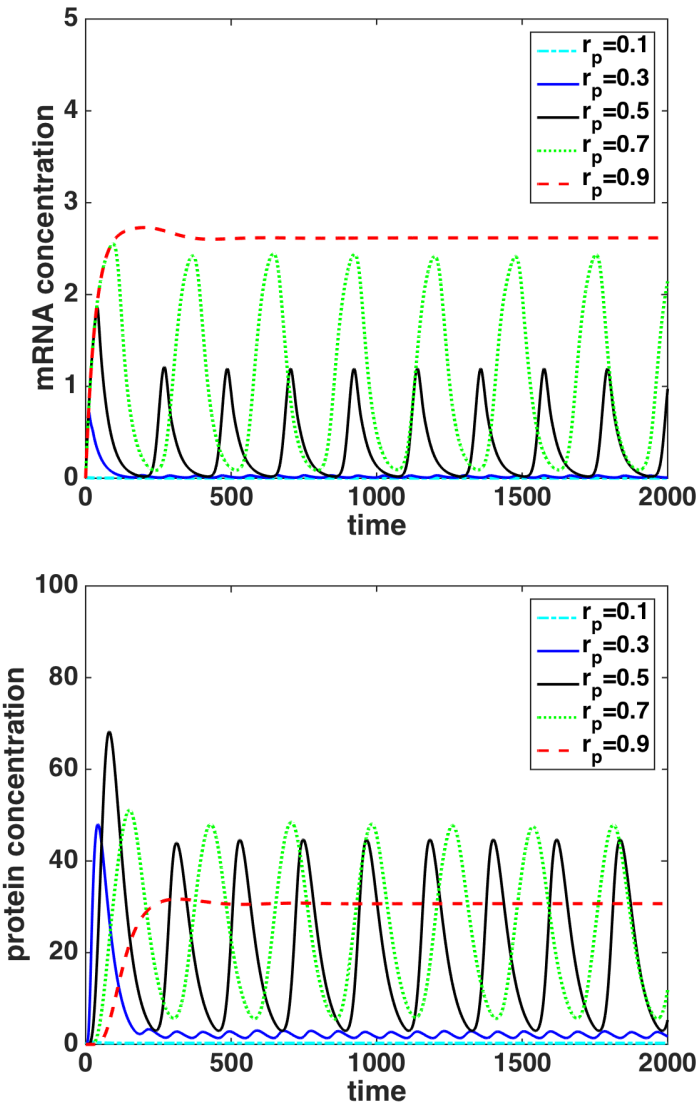

Figure B.22: Total mRNA (top panel) and protein (bot- 1269 tom panel) concentrations for the Hes1 system (B.1) in ${ }^{1270}$ 3D with spherical geometry. Shown is the behaviour ${ }^{1271}$ when the mRNA gene-site is located at $r_{m}=0.0$ and the ${ }_{1273}$ protein production zones, $r_{p}$, vary (see legend). 770
77
7
7

Eliaš, J., Dimitrio, L., Clairambault, J., Natalini, R., 2014a. Model- ${ }^{1278}$ ling p53 dynamics in single cells: physiologically based ode and ${ }^{1279}$ reaction-diffusion pde models. Phys. Biol. 11, 045001.

Eliaš, J., Dimitrio, L., Clairambault, J., Natalini, R., 2014b. The ${ }^{1281}$ p53 protein and its molecular network: modelling a missing link ${ }^{1282}$ between dna damage and cell fate. BBA Proteins Proteom. 1844, ${ }^{1283}$ 232-247.

Elowitz, M. B., Leibler, S., 2000. A synthetic oscillatory network of ${ }^{1285}$ transcriptional regulators. Nature 403, 335-338.

Geva-Zatorsky, N., Rosenfeld, N., Itzkovitz, S., Milo, R., Sigal, A., ${ }^{1287}$ Dekel, E., Yarnitzky, T., Liron, Y., Polak, P., Lahav, G., Alon, U., ${ }^{1288}$ 2006. Oscillations and variability in the p53 system. Mol. Syst. ${ }^{1289}$ Biol. 2 (2006.0033).

Glass, L., Kauffman, S. A., 1970. Co-operative components, spatial ${ }^{1291}$ localization and oscillatory cellular dynamics. J. Theor. Biol. 34, ${ }^{1292}$ 219-237.

Goodwin, B. C., 1965. Oscillatory behaviour in enzymatic control ${ }^{1294}$ processes. Adv. Enzyme Regul. 3, 425-428.

Griffith, J. S., 1968. Mathematics of cellular control processes. i. neg- ${ }^{1296}$ ative feedback to one gene. J. Theor. Biol. 20, 202-208. 1297 Hiersemenzel, K., Brown, E. R., Duncan, R. R., 2013. Imaging large ${ }^{1298}$ cohorts of single ion channels and their activity. Front Endocrinol 4 (114).

Hilbert, L., Albrecht, D., Mackey, M. C., 2011. Small delay, big waves: a minimal delayed negative feedback model captures escherichia coli single cell sos kinetics. Mol. Biosyst. 7 (9), 25992607.

Hirata, H., Yoshiura, S., Ohtsuka, T., Bessho, Y., Harada, T., Yoshikawa, K., Kageyama, R., 2002. Oscillatory expression of the bhlh factor hes 1 regulated by a negative feedback loop. Science 298 , 840-843.

Imayoshi, I., Kageyama, R., 2014. Oscillatory control of bhlh factors in neural progenitors. Trends Neurosci. 37, 531-538.

Jensen, M. H., Sneppen, J., Tiana, G., 2003. Sustained oscillations and time delays in gene expression of protein hes1. FEBS Lett. 541, 176-177.

Kageyama, R., Ohtsuka, T., Kobayashi, T., 2007. The hes gene family: repressors and oscillators that orchestrate embryogenesis. Development 134, 1243-51.

Lahav, G., Rosenfeld, N., Sigal, A., Geva-Zatorsky, N., Levine, A. J., Elowitz, M. B., Alon, U., 2004. Dynamics of the p53-mdm2 feedback loop in individual cells. Nature Genet. 36, 147-150.

Lewis, J., 2003. Autoinhibition with transcriptional delay: A simple mechanism for the zebrafish somitogenesis oscillator. Curr. Bio. 13, 1398-1408.

Mackey, M. C., Glass, L., 1977. Oscillation and chaos in physiological control systems. Science 197, 287-289.

Mackey, M. C., Santillán, M., Tyran-Kamińska, M., Zeron, E. S., 2015. The utility of simple mathematical models in understanding gene regulatory dynamics. In Silico Biol. 12 (1-2), 23-53.

Mahaffy, J. M., 1988. Genetic control models with diffusion and delays. Math. Biosci. 90, 519-533.

Mahaffy, J. M., Pao, C. V., 1984. Models of genetic control by repression with time delays and spatial effects. J. Math. Biol. 20, 39-57.

Manley, S., Gillette, J. M., Patterson, G. H., Shroff, H., Hess, H. F., Betzig, E., Lippincott-Schwartz, J., 2008. High-density mapping of single-molecule trajectories with photoactivated localization microscopy. Nat Methods 5, 155-157.

Miller, C. C., 1924. The stokes-einstein law for diffusion in solution. Proc. R. Soc. Lond. A 106, 724-749.

Momiji, H., Monk, N. A. M., 2008. Dissecting the dynamics of the hes 1 genetic oscillator. J. Theor. Biol. 254, 784-798.

Monk, N. A. M., 2003. Oscillatory expression of hes1, p53, and nf- $\kappa \mathrm{b}$ driven by transcriptional time delays. Curr. Biol. 13, 1409-1413.

Naqib, F., Quail, T., Musa, L., Vulpe, H., Nadeau, J., Lei, J., Glass, L., 2012. Tunable oscillations and chaotic dynamics in systems with localized synthesis. Phys. Rev. E 85, 046210.

Nelson, D. E., Ihekwaba, A. E. C., Elliott, M., Johnson, J. R., Gibney, C. A., Foreman, B. E., Nelson, G., See, V., Horton, C. A., Spiller, D. G., Edwards, S. W., McDowell, H. P., Unitt, J. F., Sullivan, E., Grimley, R., Benson, N., Broomhead, D., Kell, D. B., White, M. R. H., 2004. Oscillations in nf-.b signaling control the dynamics of gene expression. Science 306, 704-708.

O’Brien, E. L., Itallie, E. V., Bennett, M. R., 2012. Modeling synthetic gene oscillators. Math. Biosci. 236, 1-15.

Oeffinger, M., Zenklusen, D., 2012. To the pore and through the pore: a story of mrna export kinetics. BBA Gene Regul. Mech. 1819 (6), 494-506.

Purcell, O., Savery, N. J., Grierson, C. S., di Bernardo, M., 2010. A comparative analysis of synthetic genetic oscillators. J. R. Soc. Interface 7, 1503-1524.

Shymko, R. M., Glass, L., 1974. Spatial switching in chemical reactions with heterogeneous catalysis. J. Chem. Phys. 60, 835-841.

Smolen, P., Baxter, D. A., Byrne, J. H., 1999. Effects of macromolecular transport and stochastic fluctuations on the dynamics of genetic regulatory systems. Am. J. Physiol. 277, C777-C790. 
Smolen, P., Baxter, D. A., Byrne, J. H., 2001. Modeling circadian oscillations with interlocking positive and negative feedback loops. J. Neurosci. 21, 6644-6656.

Smolen, P., Baxter, D. A., Byrne, J. H., 2002. A reduced model clarifies the role of feedback loops and time delays in the drosophila circadian oscillator. Biophys. J. 83, 2349-2359.

Spiller, D. G., Wood, C. D., Rand, D. A., White, M. R. H., Jun 2010 Measurement of single-cell dynamics. Nature 465 (7299), 736-45. Strelkowa, N., Barahona, M., 2010. Switchable genetic oscillator operating in quasi-stable mode. J. R. Soc. Interface 7 (48), 10711082.

Sturrock, M., Hellander, A., Matzavinos, A., Chaplain, M. A. J., 2013. Spatial stochastic modelling of the hes 1 gene regulatory network: intrinsic noise can explain heterogeneity in embryonic stem cell differentiation. J. R. Soc. Interface 10, 20120988.

Sturrock, M., Murray, P. J., Matzavinos, A., Chaplain, M. A. J., 2015. Mean field analysis of a spatial stochastic model of a gene regulatory network. J. Math. Biol. 71, 921-959.

Sturrock, M., Terry, A. J., Xirodimas, D. P., Thompson, A. M., Chaplain, M. A. J., 2011. Spatio-temporal modelling of the hes1 and p53-mdm2 intracellular signalling pathways. J. Theor. Biol. 273, 15-31.

Sturrock, M., Terry, A. J., Xirodimas, D. P., Thompson, A. M., Chaplain, M. A. J., 2012. Influence of the nuclear membrane, active transport, and cell shape on the hes1 and p53-mdm2 pathways: insights from spatio-temporal modelling. Bull. Math. Biol. 74, 15311579.

Szymańska, Z., Parisot, M., Lachowicz, M., 2014. Mathematical modeling of the intracellular protein dynamics: The importance of active transport along microtubules. J. Theor. Biol. 363, 118-128.

Terry, A. J., Sturrock, M., Dale, J. K., Maroto, M., Chaplain, M. A. J., 2011. A spatio-temporal model of notch signalling in the zebrafish segmentation clock: conditions for synchronised oscillatory dynamics. PLoS One 6 (2), e16980.

Tiana, G., Jensen, M. H., Sneppen, K., 2002. Time delay as a key to apoptosis induction in the p53 network. Eur. Phys. J. B 29, 135140

van de Linde, S., Löschberger, A., Klein, T., Heidbreder, M., Wolter, S., Heilemann, M., Sauer, M., 2011. Direct stochastic optical reconstruction microscopy with standard fluorescent probes. Nat Protocols 6, 991-1009.

Won, S., Lee, B.-C., Park, C.-S., 2011. Functional effects of cytoskeletal components on the lateral movement of individual bkca channels expressed in live cos-7 cell membrane. FEBS Lett. 585, 2323-2330.

Yildirim, N., Mackey, M. C., 2003. Feedback regulation in the lactose operon: A mathematical modeling study and comparison with experimental data. Biophys. J. 84, 2841-2851.

Yordanov, B., Dalchau, N., Grant, P. K., Pedersen, M., Emmott, S., Haseloff, J., Phillips, A., 2014. A computational method for automated characterization of genetic components. ACS Synth. Biol. 3, 578-588. 